\title{
Research Paper \\ Effect of Social Competence Training on Tendency Towards High-Risk Be- haviors in Male Adolescents Living in Welfare Boarding Centers
}

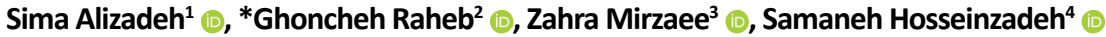 \\ 1- M.A. Student of Social Work, Department of Social Work, University of Social Welfare and Rehabilitation Sciences, Tehran, Iran \\ 2. Assistant Professor, Department of Social Work, University of Social Welfare and Rehabilitation Sciences, Tehran, Iran. \\ 3. Ph.D. Student of of Social Work, Department of Social Work, University of Social Welfare and Rehabilitation Sciences, Tehran, Iran \\ 4. Assistant Professor, Department of Statistics, University of Social Welfare and Rehabilitation Sciences, Tehran, Iran.
}

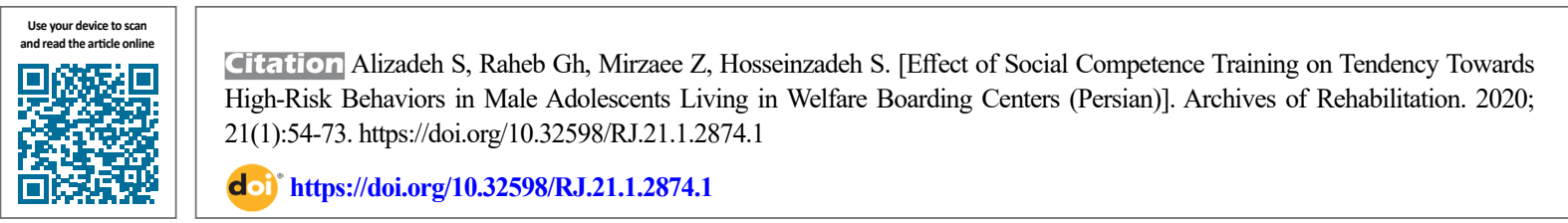

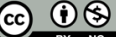

Received: 24 Sep 2018 Accepted: 24 Jun 2019 Available Online: 01 Apr 2020

Keywords:

Social skills, Ado-

lescent, Health Risk

Behaviors

\section{A B STRACT}

Objective High-risk behaviors are seriously threatening health and wellbeing, and increasing the likelihood of physical, psychological, and social consequences. High-risk behaviors, such as tobacco use, drug abuse, alcohol use, or malicious behaviors can endanger the health and wellbeing of adolescents and hinder their success and future growth. Today, the prevalence of high-risk behaviors among adolescents has become one of the most important concerns in society and despite the provisions made in recent years, its rate has increased. One of the vulnerable groups is orphaned/abandoned adolescents who face various social and psychological problems during their stay in welfare bearding centers that requires special skills to deal with. This study aimed to investigate the effect of social competence training on the tendency towards high-risk behaviors in male adolescents living in welfare boarding centers.

Materials \& Methods This is a quasi-experimental study with a pre-test-post-test design. The study population included all male adolescents aged 12-18 years living in welfare boarding centers located in Sabzevar and Mashhad cities in Iran. For sampling, 15 adolescents were selected from a boarding center in Sabzevar City by census method and assigned to the experimental group, and 15 from a boarding center in Mashhad City selected randomly and assigned as controls. The experimental group received social competence training program in twelve 60-min sessions, while the control group received only two 8-hour first aid training. Iranian Adolescents Risk-Taking Scale (IARS) and a demographic form were used to collect data before and after the intervention. The collected data were analyzed by Multivariate Covariance Analysis (MANOVA) in SPSS V. 22. Results The mean IARS scores in the experimental group decreased from 93.9 to 86.81 after the intervention, and the t test results indicated a significant difference between the mean scores before and after the intervention ( $t=2.97, P=0.010)$. The MANCOVA results revealed a significant difference between the mean overall score of IARS ( $F=24.08, P<0.001)$, and between the mean scores of IARS dimensions (tendency towards dangerous driving, violence, smoking, drug abuse, alcohol use, sexual activity and behavior, and relationship with the opposite gender) in the experimental and control groups after the intervention $(P<0.001)$. Based on Eta squared values, $47.1 \%$ of changes in the tendency towards high-risk behaviors in subjects can be explained by the social competence training program. Therefore, social competence training had a positive effect on reducing the tendency towards risky behaviors among male adolescents living in welfare boarding centers. Conclusion The social competence training program could improve the skills and adaptive capacity of orphaned/abandoned adolescents in different situations to deal with their problems. Therefore, it is recommended that social competence training programs be provided for male adolescents living in boarding schools to effectively increase their four social competence skills and ultimately reduce their tendency to engage in high-risk behaviors.

\section{* Corresponding Author:}

Ghoncheh Raheb, PhD.

Address: Department of Social Work, University of Social Welfare and Rehabilitation Sciences, Tehran, Iran.

Tel: +98 (21) 22180084

E-Mail: ghraheb@gmail.com 


\section{Introduction}

dolescence is a critical time of life. Behavioral problems peak during adolescence and 1 youth periods. According to the 2016 population census, about 6 million people in Iran are 14-19 years old. Since Iran's population is young and many adolescents are unprepared in dealing with challenging issues, the social and psychological health of adolescents as vulnerable a group is one of the main priorities of the country. Meanwhile, orphaned and abandoned adolescents living in daycare centers facing family issues and having additional problems compared with other adolescents. One of these problems is their high-risk behaviors.

High-risk behaviors include smoking, drug abuse, alcohol use, dangerous driving, and early sexual activity. These behaviors endanger the health and wellbeing of adolescents, young people, and others in the community. The results of a 2015 US national survey showed that many high school students are at risk for high-risk behaviors that are the leading cause of death among people aged 10-24 years in the United States. Research in Iran also shows the prevalence of high-risk behaviors such as smoking and using hookah, alcohol, and drugs among adolescents aged 14-19 years. It is important to address the issue of high-risk behaviors because they cause personal and social problems and accident-related deaths.

High-risk behaviors have several determinants at different levels such as family, peers, school, and some individual and social competence. Therefore, considering the importance of the family in the development of the individual, it can be said that abandoned and orphaned children and adolescents grow up with various behavioral and emotional problems due to lack of enough social/emotional experience. Moreover, the study of some characteristics and problems of orphaned and abandoned children and adolescents compared with the children living in the family suggests that the occurrence of emotional and behavioral disorders and the tendency towards high-risk behaviors is related to lack of social/emotional competence. Thus, social competence training has been proposed by experts in this field.

According to Felner et al. (1990), social competence has four dimensions: cognitive, behavioral, emotional, and motivational skills [20]. Some studies have been conducted on social competence training at different ages and fields. Rockhill et al. (2009) examined the role of social competence in high school students. Their results showed that social competence training reduces aggression, anxiety, de- pression, social maladaptation, and antisocial behavior of adolescents [21].

Ferreira et al. (2012) in a study evaluated the role of social and emotional competence on risky behaviors in adolescence. Their research emphasized characteristics such as empathy, cooperation, goals, and aspirations as intermediaries between social and emotional competence, substance use, tobacco use, and alcohol use. Their findings showed a significant relationship between the social and emotional competence of adolescents and the reduction of their highrisk behaviors [6].

Langeveld et al. (2012) and Gundersen (2010) in their study on behavioral problems in children and adolescents, found that increased social competence by reducing factors such as self-control, anger management, cooperation, and empathy could reduce their behavioral problems [22, 23]. Kim et al. (2018) also reported similar results [24]. Haight et al. (2010) examined the effect of mental health intervention on children and adolescents aged 7-15 years living in boarding schools. Their results showed that the experimental group which received social work intervention had a significant improvement in mental health and some behaviors compared with the control group [25].

Based on the literature, many studies have been conducted on the effectiveness of social competence and its related skills separately in normal and abandoned/orphaned adolescents. However, few have studied the effectiveness of social competence training on risky behaviors of abandoned/ orphaned adolescents. In this regard, this study aimed to investigate the effect of teaching four dimensions of social competence on the tendency to high-risk behaviors of abandoned/orphaned male adolescents living in welfare boarding centers affiliated to the Iranian Welfare Organization.

\section{Materials and Methods}

This is a quasi-experimental study with a pre-test-post-test design. The study population consists of all male adolescents living in welfare boarding centers located in Sabzevar and Mashhad cities, Iran. There was only one welfare boarding center in Sabzevar named Sobhan, which took care of 15 abandoned/orphaned male adolescents aged 12-18 years. All of them were entered into the study and assigned to the experimental group. For the control group, the adolescents in Golestan-E Ali center located in Mashhad City were selected based on a convenience sampling method. There were 29 boys in this center. Of these, 15 who had met inclusion criteria were randomly selected and assigned as controls. 
Table 1. The social competence training protocol used in the study

\begin{tabular}{|c|c|c|}
\hline Domain & Session & Content \\
\hline \multirow{5}{*}{ Cognitive skills } & 1 & Introduction and getting acquainted \\
\hline & 2 & Self-awareness skills \\
\hline & & \\
\hline & 3 & Distinguishing positive beliefs from negative beliefs and how to deal with them \\
\hline & 4 & Problem-solving and decision-making skills \\
\hline \multirow{3}{*}{ Behavioral skills } & 5 & Communication skills \\
\hline & 6 & How to be assertive \\
\hline & 7 & How to acquire social support \\
\hline \multirow{2}{*}{ Emotional skills } & 8 & Identifying positive and negative emotions, emotional coping skills, stress contro \\
\hline & 9 & Anger management \\
\hline \multirow{3}{*}{ Motivational skills } & 10 & Self-efficacy skills \\
\hline & 11 & Continuation of the previous session \\
\hline & 12 & Summarizing, conclusion, and post-test phase \\
\hline
\end{tabular}

The inclusion criteria were being 12-18 years old, willing to participate in the study, having a normal state of consciousness, lacking a history of mental illness, and living in the boarding centers for at least one year. The exclusion criteria were unwillingness to continue participation, transfer, or discharge from the center.

Data collection tools were a demographic form and Iranian adolescents risk-taking scale (IARS) developed by Zadeh Mohamadi et al. (2008) [30]. It has 38 items rated based on a 5-point Likert-type scale from 1= completely disagree to $5=$ completely agree. Before distributing the questionnaires, the purpose of the study was explained to the participants and they were assured of the confidentiality of their information. Then, informed consent was obtained from them. After determining the time of the classes, according to the previous plan, the social competence training package was given to the intervention group for 2 months in 12 sessions, while the control group received only first aid training. Table 1 lists the content of the training sessions.

The intervention protocol was according to the social competence model of Felner (1990) and prepared by researchers under the supervision and approval of qualified faculty members [20]. The questionnaires were completed before and after the intervention. One month after the intervention, the tendency towards high-risk behaviors was compared between the two groups. The collected data were analyzed in SPSS V. 22 by using multivariate ANCOVA, independent $t$ test, paired $t$ test, Shapiro-Wilk test, and Levene's test.

\section{Results}

The mean age of the control group was 14.40 years and of the experimental group 14.47 years. Most study participants had a middle school education. In the experimental group, most boys were abandoned $(3.73 \%$ ); while in the control group, all three types of bad supervisions were equally distributed (33\%). Also, most of them had entered welfare boarding centers from childhood. The Shapiro-Wilk test was used to check whether the distribution of variables was normal. The results showed a normal distribution before and after the intervention $(\mathrm{P}>0.05)$. The mean and standard deviation of the scores of a tendency towards high-risk behaviors and its subscales for the experimental and control groups are presented in Table 2. As can be seen, post-test scores decreased compared with the pre-test scores.

According to the results shown in Table 3, the intervention had a significant effect on the post-test scores of IARS and its dimensions $(\mathrm{P}<0.05)$. Based on Eta squared values, it could explain $40 \%, 23.8 \%, 20.2 \%, 18.1 \%, 13.3 \%, 29.2 \%$, and $20.9 \%$ of changes in the tendency to dangerous driving, violence, smoking, drug abuse, alcohol use, sexual behavior, and the opposite sex, respectively. Results of multivari- 
Table 2. The mean IARS scores of participants in both groups

\begin{tabular}{|c|c|c|c|}
\hline \multirow{3}{*}{ Dimensions } & & \multicolumn{2}{|c|}{ Mean $\pm S D$} \\
\hline & & \multicolumn{2}{|c|}{ Groups } \\
\hline & & Experimental & Control \\
\hline \multirow{2}{*}{ Dangerous driving } & Pre-test & $19.86 \pm 4.58$ & $16.86 \pm 4.12$ \\
\hline & Post-test & $13.33 \pm 3.88$ & $17.86 \pm 4.77$ \\
\hline \multirow{2}{*}{ Violence } & Pre-test & $14.33 \pm 4.76$ & $12.93 \pm 4.19$ \\
\hline & Post-test & $11.53 \pm 2.16$ & $13.33 \pm 3.59$ \\
\hline & Pre-test & $11.06 \pm 5.78$ & $11.53 \pm 4.80$ \\
\hline \multicolumn{4}{|l|}{ Smoking } \\
\hline & Post-test & $8.80 \pm 2.75$ & $12.26 \pm 4.81$ \\
\hline & Pre-test & $16.33 \pm 7.97$ & $17.86 \pm 6.44$ \\
\hline \multicolumn{4}{|l|}{ Drug abuse } \\
\hline & Post-test & $15 \pm 3.74$ & $18.66 \pm 5.57$ \\
\hline & Pre-test & $15.40 \pm 8$ & $14 \pm 4.56$ \\
\hline \multicolumn{4}{|l|}{ Alcohol use } \\
\hline & Post-test & $14.13 \pm 4.88$ & $15.73 \pm 5.17$ \\
\hline \multirow{3}{*}{ Sexual behavior } & Pre-test & $13.26 \pm 5.88$ & $11.33 \pm 3.24$ \\
\hline & & & \\
\hline & Post-test & $10.80 \pm 3.94$ & $12.26 \pm 3.49$ \\
\hline \multirow{2}{*}{$\begin{array}{l}\text { A tendency to the opposite } \\
\text { sex }\end{array}$} & Pre-test & $10.86 \pm 5.57$ & $9.73 \pm 4.49$ \\
\hline & Post-test & $8.26 \pm 3.12$ & $10.66 \pm 3.86$ \\
\hline & Pre-test & $100.93 \pm 32.57$ & $94.26 \pm 22.60$ \\
\hline \multicolumn{4}{|l|}{ Total } \\
\hline & Post-test & $81.86 \pm 16.07$ & $100.80 \pm 20.67$ \\
\hline
\end{tabular}

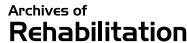

ate ANCOVA presented in Table 3 showed that the social competence training program had a significant effect on the subjects' tendency towards high-risk behaviors $(\mathrm{F}=24.08$, $\mathrm{P}<0.001)$. The Eta squared value for overall IARS score showed that $47.1 \%$ of changes in their tendency can be explained by social competence training. Hence, the main hypothesis of this study was confirmed.

\section{Discussion}

The purpose of this study was to investigate the effectiveness of social competence training on the tendency towards high-risk behaviors in adolescent boys living in welfare boarding centers in Iran. The first finding of this study was that social competence training had a positive effect on reducing the tendency towards dangerous driving. This finding is consistent with the Shults et al. (2018) study results regarding the social competence training for correcting adolescents' behaviors in driving [31], and Foreman et 
Table 3. Multivariate ANCOVA results of comparing the mean IARS score and its dimensions after the intervention

\begin{tabular}{|ccccccc}
\hline Variables & Sum of Squares & df & Mean Squares & $\mathbf{F}$ & $\mathbf{P}$ & ETA Squared \\
\hline Dangerous driving & 16.252 & 1 & 16.252 & 12.18 & $*<0.001$ & 402.0 \\
\hline Violence & 52.40 & 1 & 52.40 & 42.8 & $* 0.007$ & 238.0 \\
\hline Smoking & 16.81 & 1 & 16.81 & 81.6 & $* 015.0$ & 202.2 \\
\hline Drug abuse & 41.63 & 1 & 41.63 & 96.5 & $* 021.0$ & 181.0 \\
\hline Alcohol use & 78.43 & 1 & 78.43 & 14.4 & $* 05.0$ & 133.0 \\
\hline Sexual behavior & 72.52 & 1 & 72.52 & 11.11 & $* 002.0$ & 292.0 \\
\hline A tendency to the opposite sex & 43.60 & 1 & 43.60 & 12.7 & $* 013.0$ & 209.0 \\
\hline Total & 54.3662 & 1 & 54.3662 & 08.24 & $*<0.001$ & 471.0 \\
\hline
\end{tabular}

* Significant difference $(\mathrm{P}<0.05)$.

al. (2018) study results regarding appropriate interventions on the behavioral and cognitive dimensions of subjects for reducing dangerous driving [32].

The second finding of the study was that social competence training reduced the tendency towards violence in the subjects. This result is in agreement with the results of Van Vugt et al. (2013), Matson et al. (2009), Kõiv (2012), and DeWall et al. (2011) who indicated the use of social skills training, managing emotions and having positive relationships with peers as protective factors against behavioral problems, especially aggression [35-38].

Our results also showed that social competence training reduced the tendency toward cigarette smoking. This finding is consistent with Onyechi et al. (2017) and Margueritte et al. (2018) results. They reported the effectiveness of training on side effects and health problems caused by smoking and tobacco use and the correction of attitudes towards smoking [41, 42]. Social competence training could also reduce the tendency towards drug abuse and alcohol use. This is consistent with the findings of Khaledian et al. (2014), Bahreini-Borujeni et al. (2014), McDonald et al. (2017), Wang et al. (2018), and Mohammad-Najar et al. (2017) [46-50]. Those studies reported the effectiveness of teaching each of the components of social competence in reducing the tendency to use drugs in adolescents and addicts. Also, it is consistent with the results of Chapman et al. (2018) who stated that to prevent the early onset of alcohol and marijuana use and reduce their risk, effective prevention in adolescence through increasing awareness of their complications, and related injuries are very important [51].
Social competence training could also reduce the tendency toward sexual intercourse and behavior. This finding agrees with the results of Perez-Cuevas et al. (2016) and Jeong et al. (2017). They showed that educational intervention and increasing the awareness of high-risk sexual behaviors in students could have a positive effect on reducing their tendency toward unprotected sex and sexually transmitted diseases [54, 55]. Overall, social competence training could reduce the tendency of adolescents towards high-risk behaviors. Weiss et al. (2017) also reported that teaching social skills leads to improved mental, emotional, and behavioral health performance in students [59]. Burrus et al. (2018) in their study concluded that educational interventions for vulnerable people, especially abandoned/ orphaned adolescents living in boarding centers, can prepare them for adulthood and ultimately lead to behavioral changes for having health and long-term wellbeing and to reduce their risky behaviors [60].

Social competence is the ability of a person to deal effectively with the daily challenges of life, to try to maintain mental wellbeing, and to express it consistently and positively. Impaired social competence often causes many psychological and social problems, such as drug and alcohol abuse, delinquency, antisocial behavior, and maladaptation. In this regard, the role of the social worker as the leader of the group is of special importance. According to Bandura's theory of social learning, the behavior of social workers in teamwork is considered a pattern of behavior. Group education itself can have a positive effect on increasing adaptability, because the gathered individuals in a group and having similar problems are effective in reducing stress and the negative mood of adolescents and, thus, increasing acceptance of the problem and coping with it. 
There were some limitations in conducting this study. The study tool Iranian Adolescents Risk-Taking Scale (IARS) was a self-report instrument, so the results may not be very accurate. Limited sample size, sampling method, and problems due to the asking sensitive questions are other limitations of this research. Moreover, because the research was done using a convenience sampling method, it is recommended that the generalization of the results to the adolescents living in other welfare boarding centers should be done with caution. It is suggested that due to the increasing population of abused and orphaned children and adolescents, workshops and group education should be held to reduce their high-risk behaviors in the study welfare centers and other day/night care centers.

\section{Conclusion}

Social competence training has a significant effect on reducing the tendency towards high-risk behaviors in abandoned/orphaned adolescents. Due to insufficient positive feedback from their successful performance, they have a feeling of low competence and self-esteem, and thus are more likely to be exposed to high-risk behaviors. A social competence training program can be used as a method for reducing their tendency towards high-risk behaviors.

\section{Ethical Considerations}

\section{Compliance with ethical guidelines}

This study has ethical approval code obtained from the Research ethics Committee of University of Social Welfare and Rehabilitation Sciences. Prior to study, a written informed consent was obtained from the participants. To observe ethical principles, an educational package was also prepared and presented to the controls. Participants were assured of the confidentiality of their information.

\section{Funding}

This study was extracted from the master thesis of Ms. Sima Alizadeh approved by the Department of Social Work, University of Social Welfare and Rehabilitation Sciences.

\section{Authors' contributions}

Investigation: Sima Alizadeh; Conceptualization, editing \& review: Ghoncheh Raheb; Methodology: Zahra Mirzaei, Data analysis: Samaneh Hasanzadeh.

\section{Conflicts of interest}

The authors declared no conflict of interest.

\section{Acknowledgements}

The authors would like to thank all employees and adolescents living in boarding schools under the supervision of Welfare Organization in Sabzevar and Mashhad cities for their valuable cooperation. 
This Page Intentionally Left Blank 


\title{
اثربخشى آموزش كفايت اجتماعى بر ترايش به رفتارهاى يرخطر نوجوانان يسر مراكز شبانهروزى بهزيستى در شهرهاى مشهلد و سبزوار
}

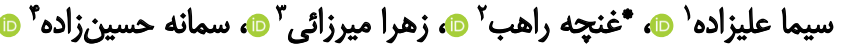

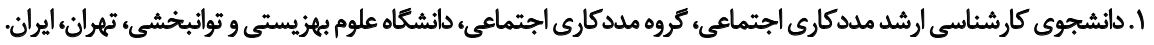

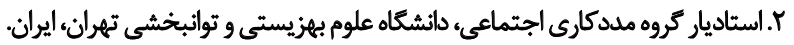

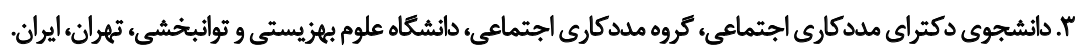

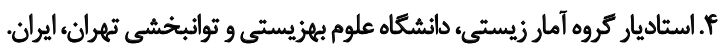

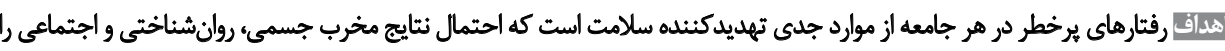

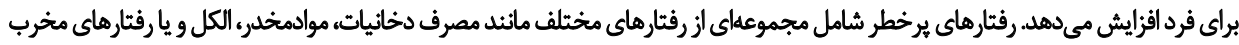

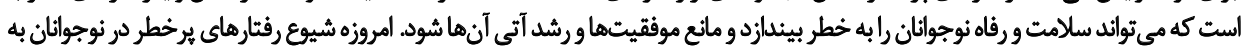

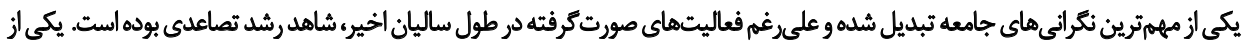

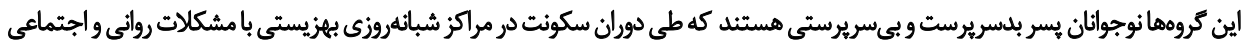

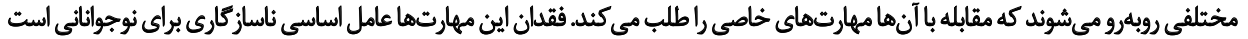

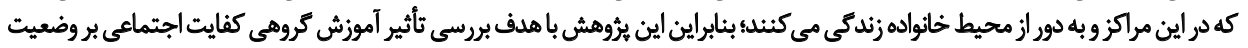

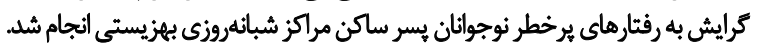

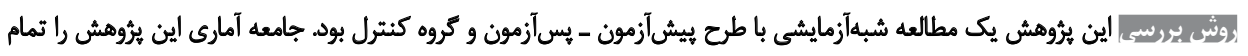

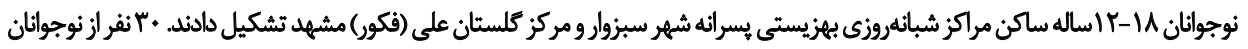

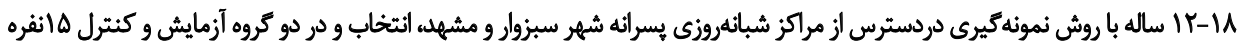

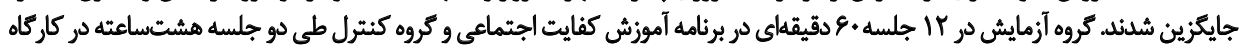

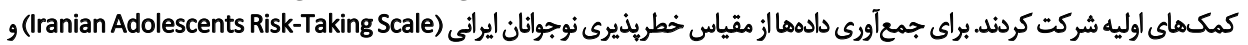

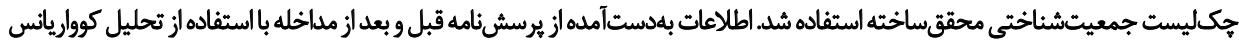

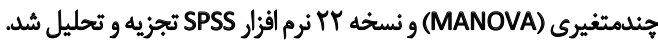

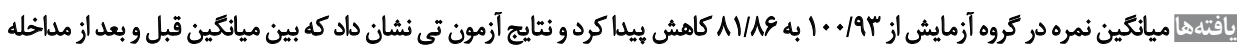

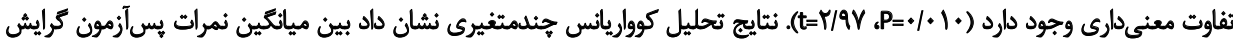

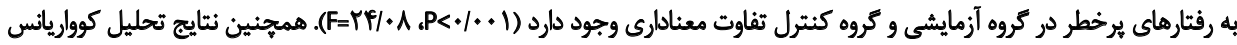

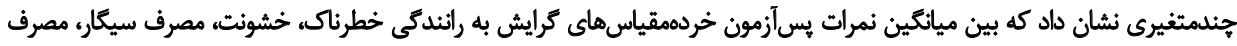

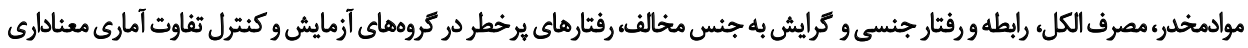

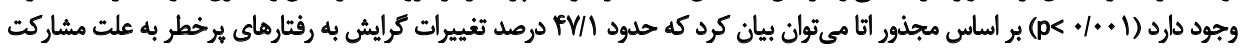

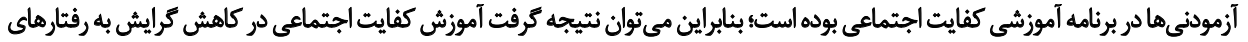

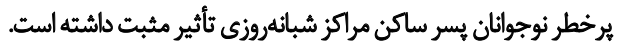

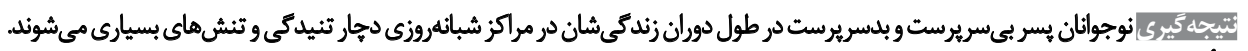

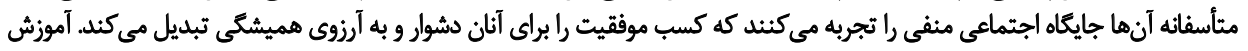

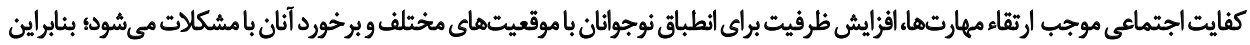

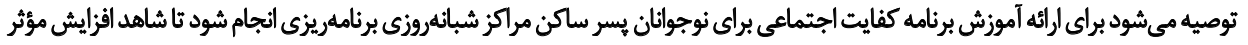

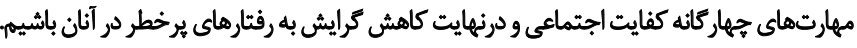

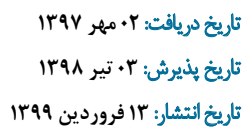


ممكن است فرد را به سمت ساير رفتار هاى خطرئاى نيز سوق

مقدمه

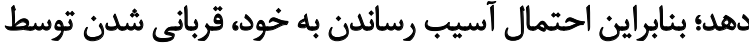

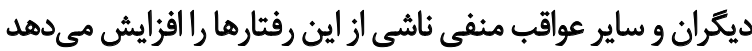

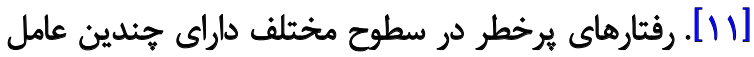

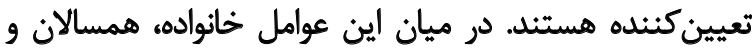

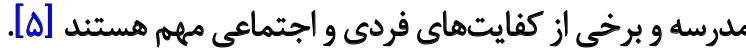
در اغلب تحقيقات، بيشتر عوامل تعيينكنينده رفتار به عنصر

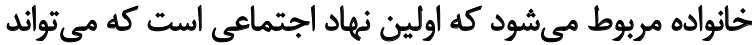

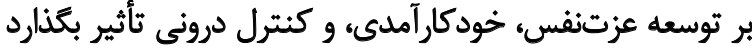

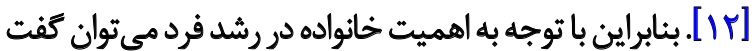

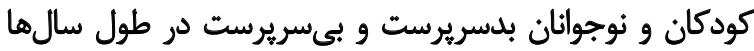

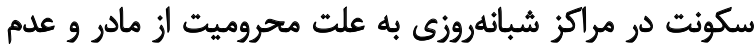

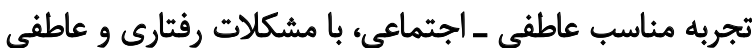

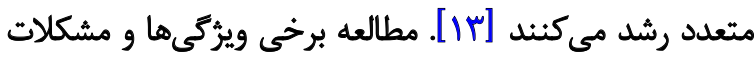

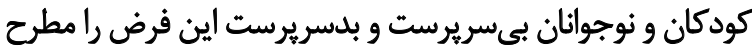

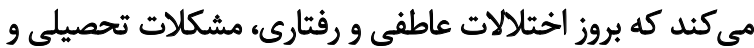

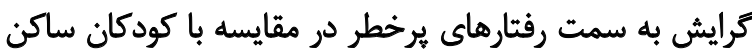

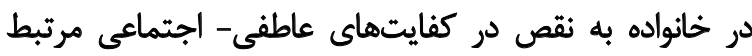

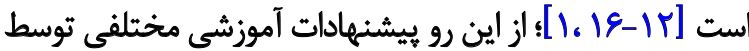

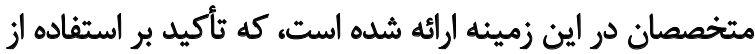

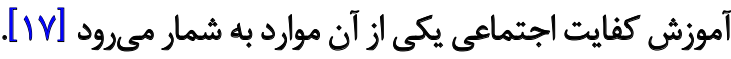

كفايت اجتماعى يكى از سازههاي اصلى بهيداشت روائى و يك

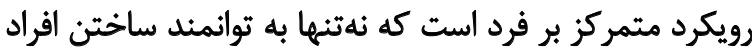

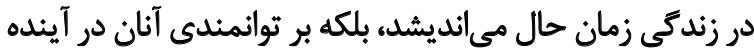

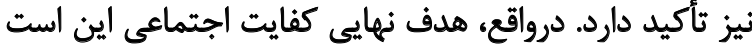

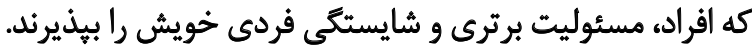

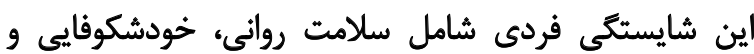

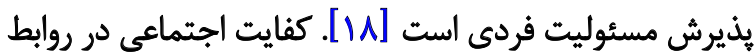

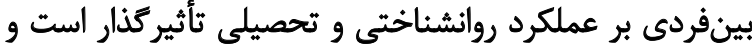

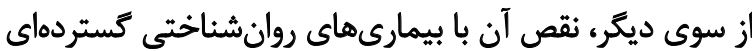

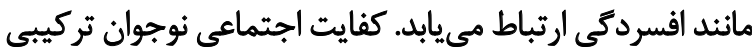

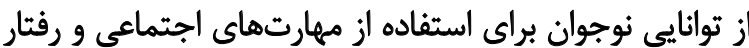

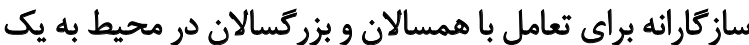

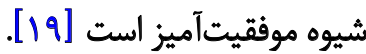

فلنر، ليس وفيلييس معتقدند كه كفايتاجتماعى جهار بُعد دارد:

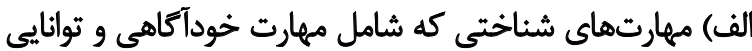

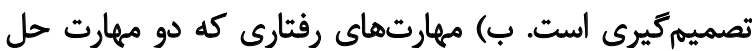

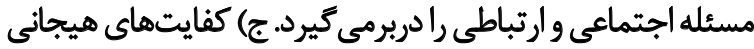

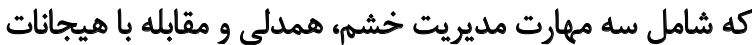

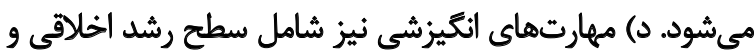

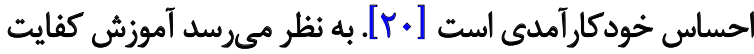

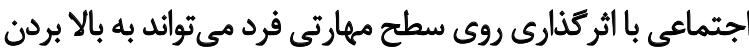

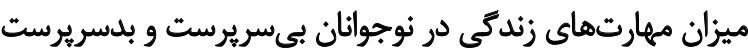

دوره نوجوانى از دورههاى بحرانى زندكى است. مشكلات رفتارى

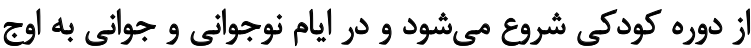

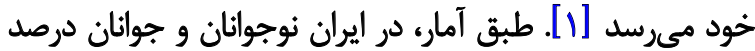

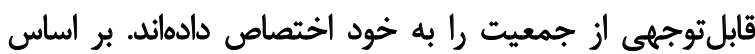

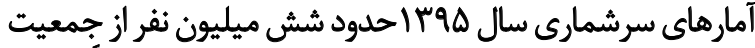

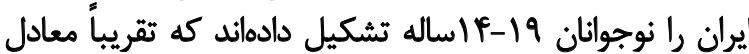

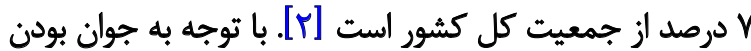

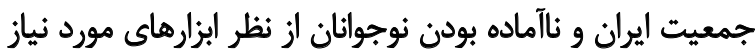

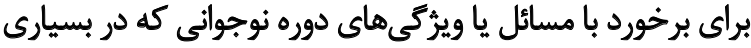

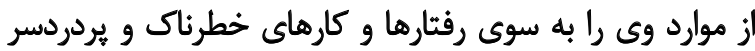

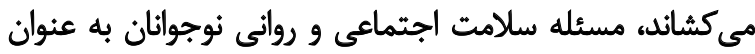

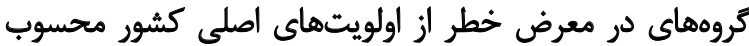

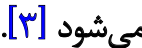

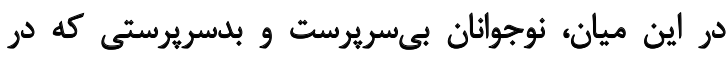

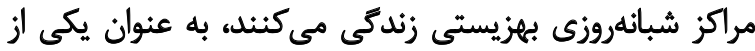

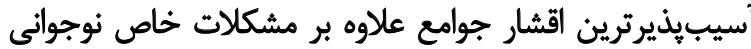

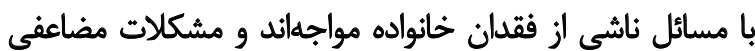

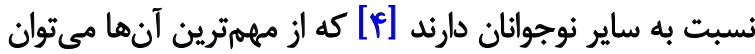

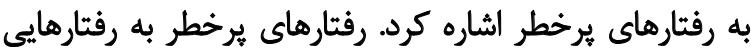

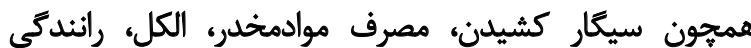

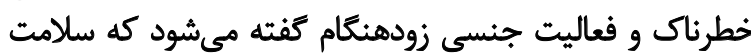

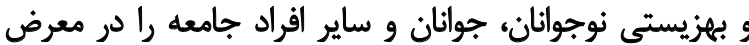

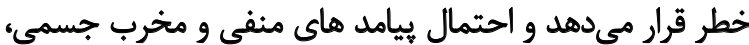

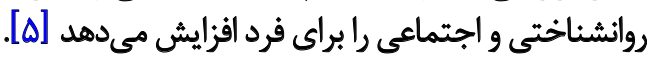

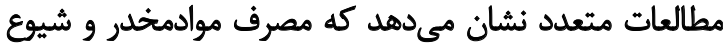

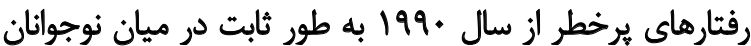

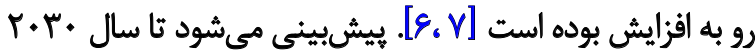

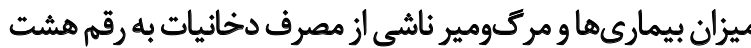

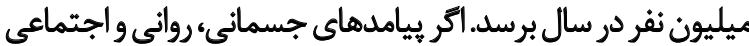

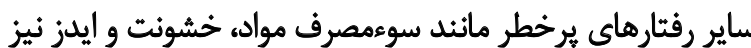

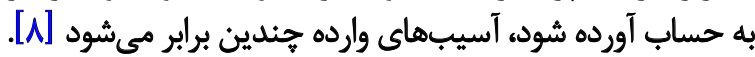

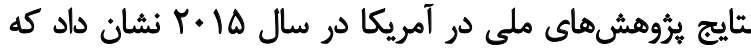

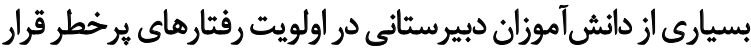

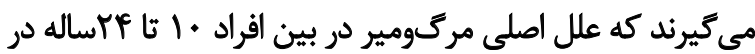

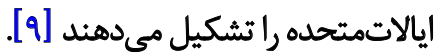
يرؤشهاى انجامشده در ايران نيز بيانكر شيوع رفتارهاى

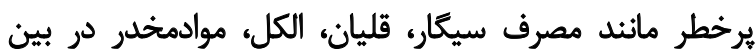

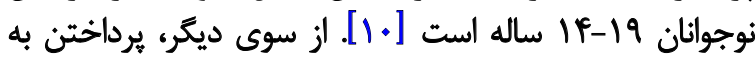

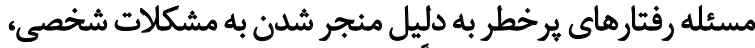

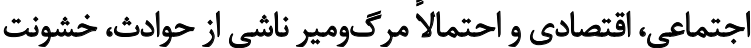

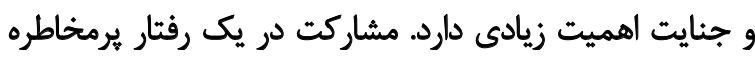


ارتباطى و خودكارآمدى در نوجوانان ساكن مراكز شبهخانوادها.

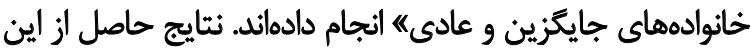

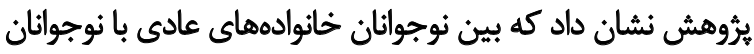

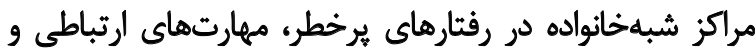

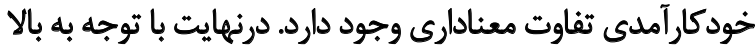

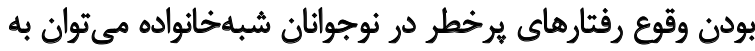

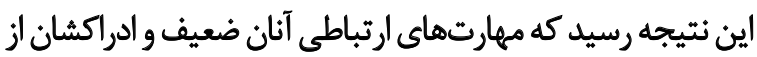

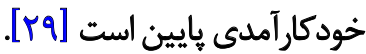

با توجه به بيشينهاى يُوروهشى يافتشده، مطالعات زيادى در

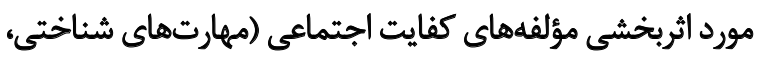

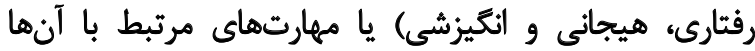

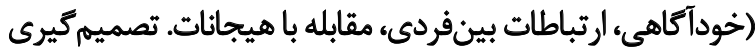

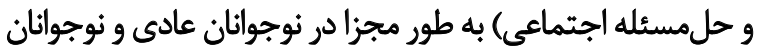

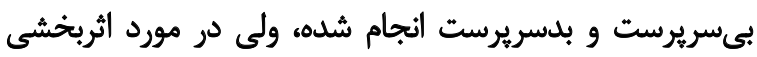

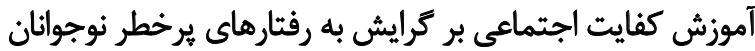

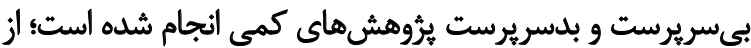

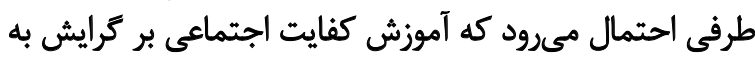

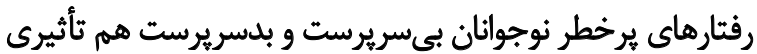

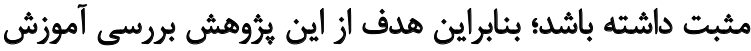

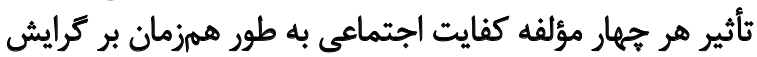

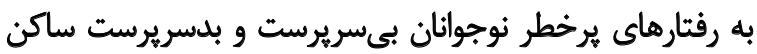

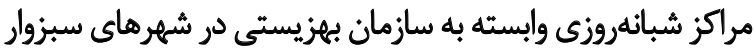

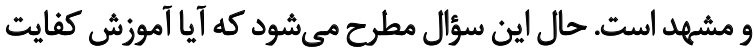

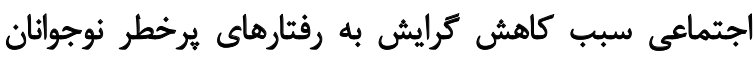
بي سريرست و بلدسريرست مي شئود؟ ووشى بوربس

اين يرؤهش از نوع شبها آزمايشى با كروه آزمايش و كنترل بود. با

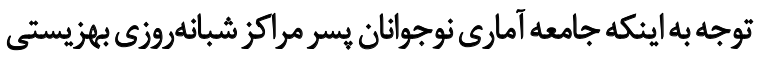

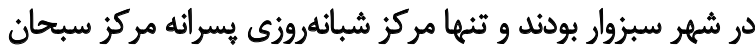

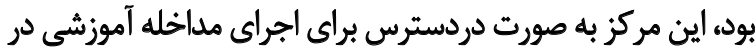

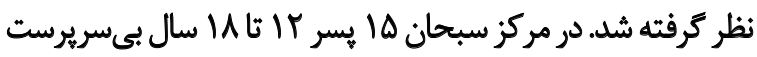

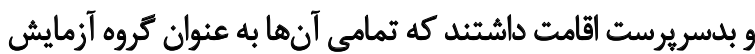

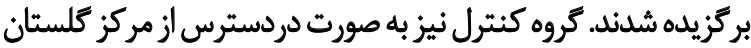

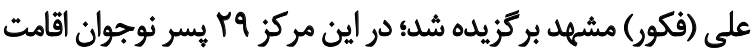

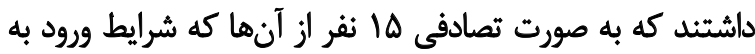

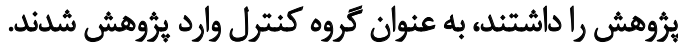
ملاكهاى ورود به فرايند مطالعه عبارت بود ازي بـان بران

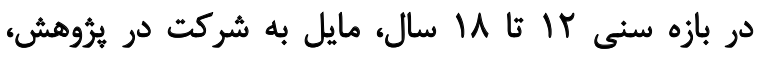

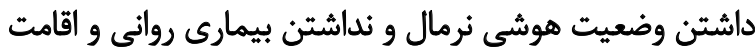

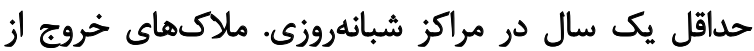

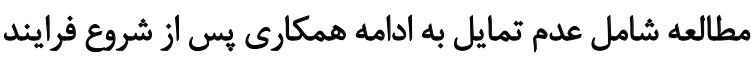
يروهش و جابهجايى يا ترخيص نوجوان از مركز بود.
براى يیشكيرى، كاهش و كنترل آسيبهاى اجتماعى كمك كند. بهرهيرى از جهار مؤلفه كفايت اجتماعى مانع تعميميذيدي

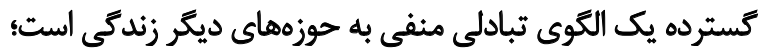

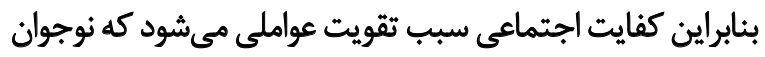

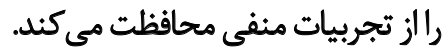

در بررسىهاى جامع، يثوهشهايى در خصوص آموزش كفايت

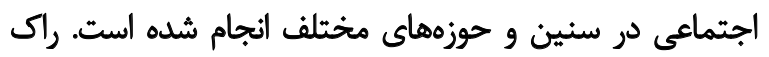
هيل و همكاران نقش كفايت اجتماعى و حمايت اجتماعى

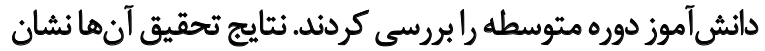

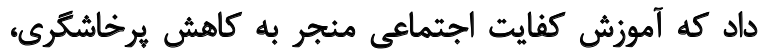

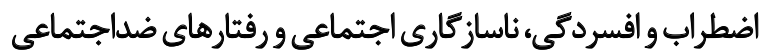

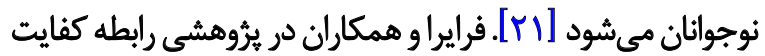

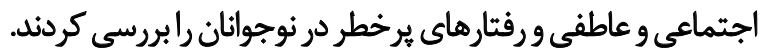

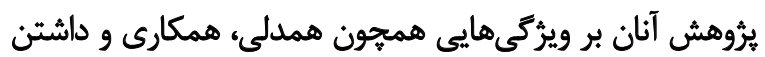

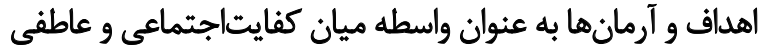

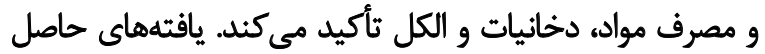

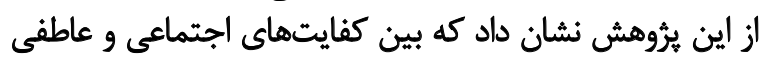

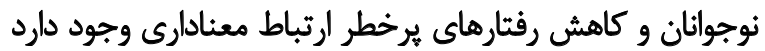

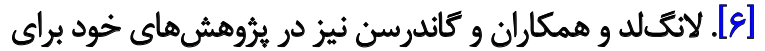

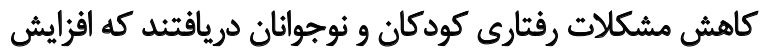

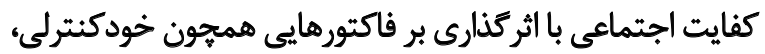

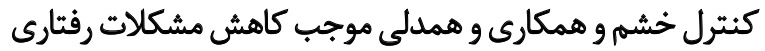

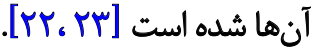

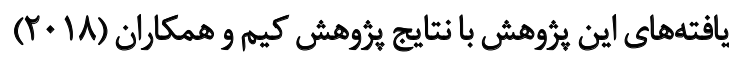

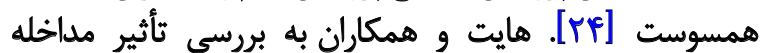

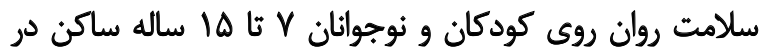

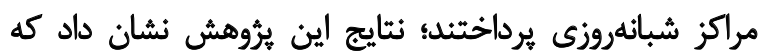

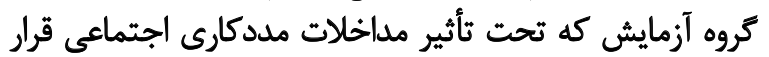

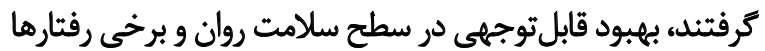

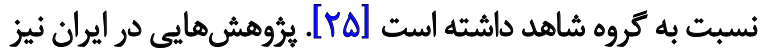

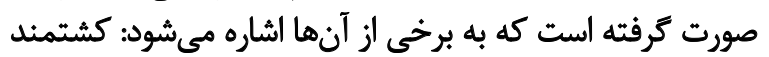

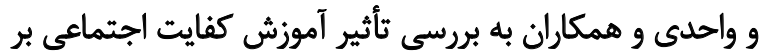

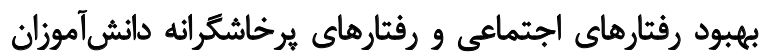

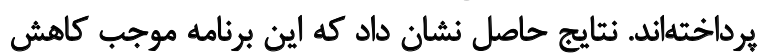

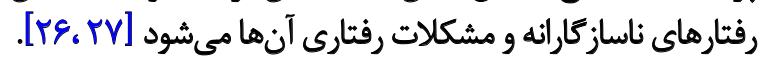
فيار احمديان تأثير آموزش كفايت جتماعى برارتقاى توانمندىهاى

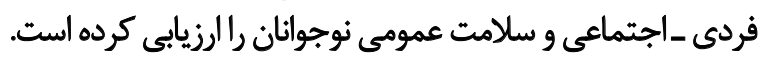

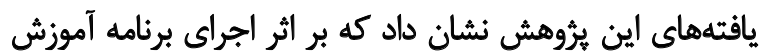

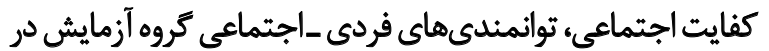

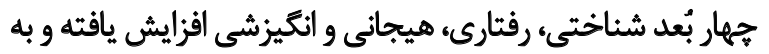

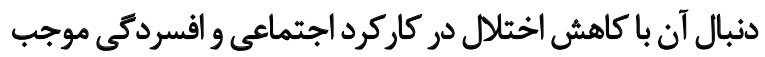

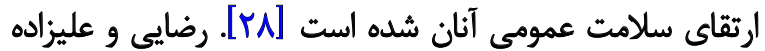

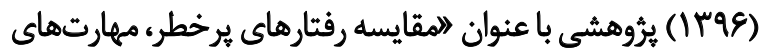


اطمينان داده شد كه اطلاعات اخذشده محرمائه خواهد ماند. برنامه مداخلهاي: برنامه مداخلهاي مبتنى بر كفايت اجتماعى

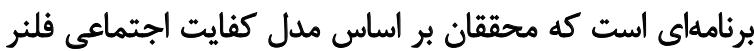

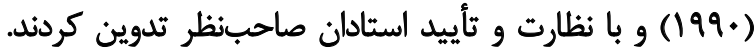

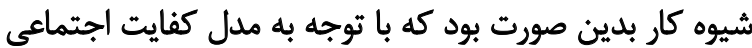

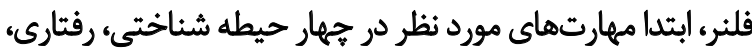

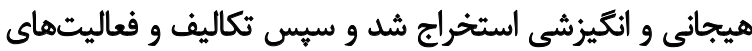

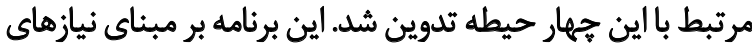

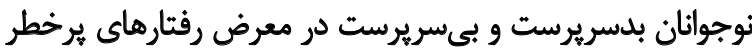

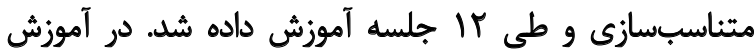

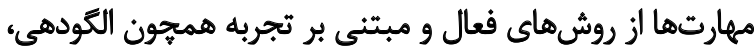
بارش مغزى، بحث، ايفاى نقش، تصحيح و بازخورد استفاده شد.

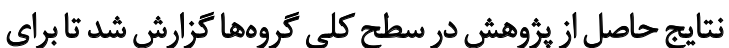

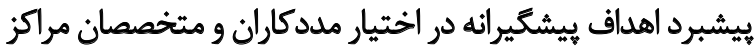

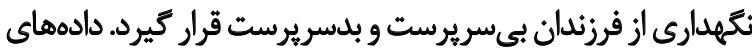

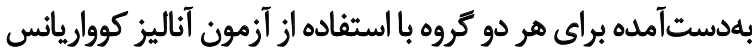

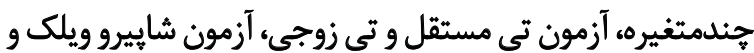

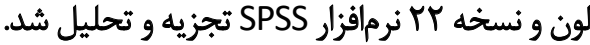

يافتهها

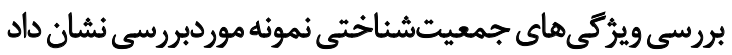

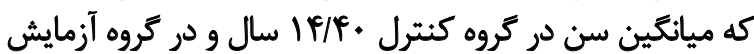

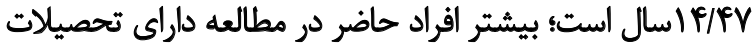

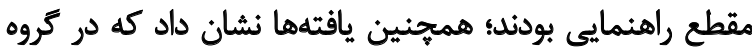

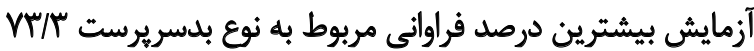

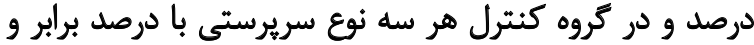

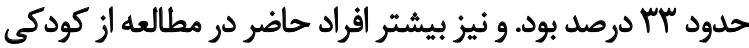

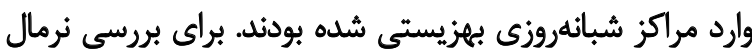

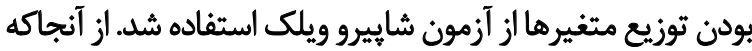

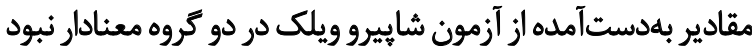

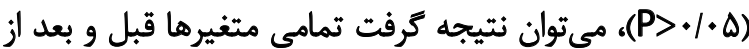
مداخله داراي توزيع نرمال بودماند.

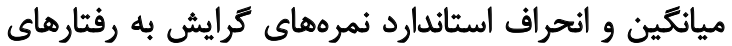

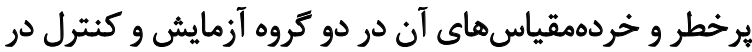

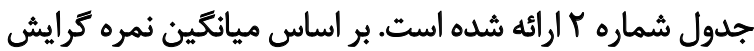

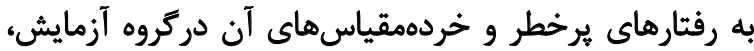

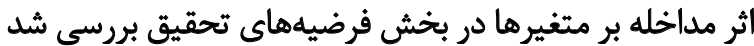

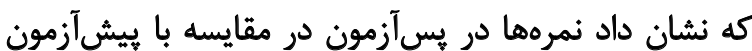

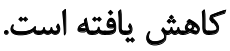

با توجه به نتايج جدول شماره با، مداخله انجامشده اثر معناداري

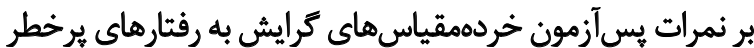

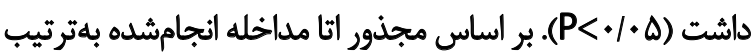

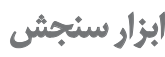

مقياس خطريذيرى نوجوانان ايرانى': اين مقياس را زاده

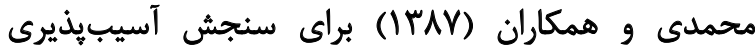

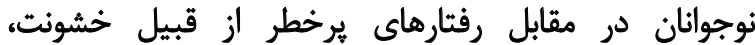

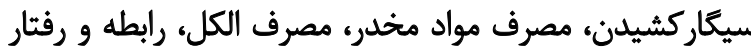

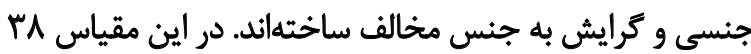

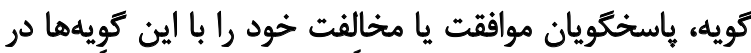

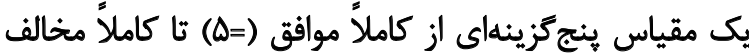

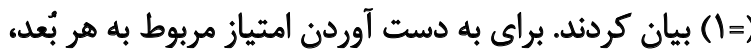

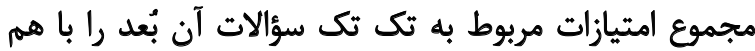

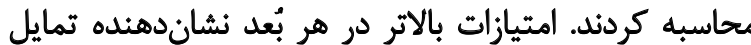

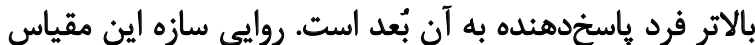

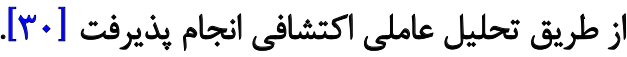
علاوهبر اين،نوجوانان به جكليستويرّكى هاى جمعيتشناختى

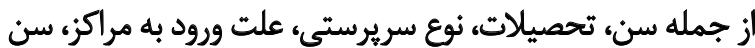
ورود به مراكز و زمان ورود به مراكز ياسخ دادئد.

روش اجراى ثيروهش: براى انجام يروهش از سازمان بهزيستى

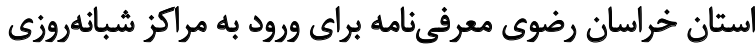

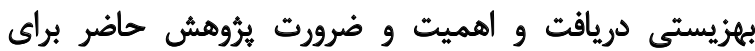

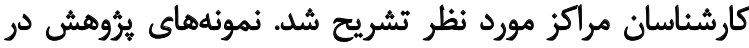

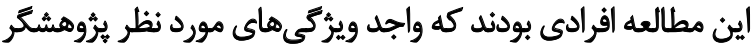

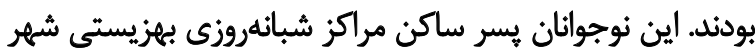

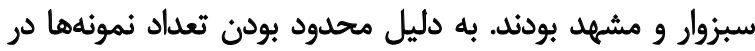

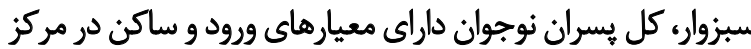

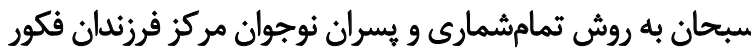

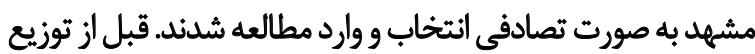

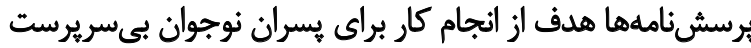

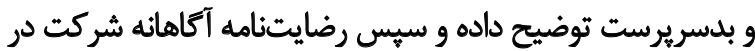

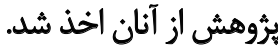

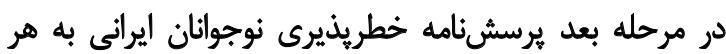

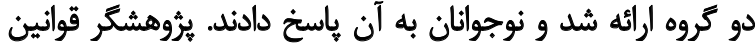

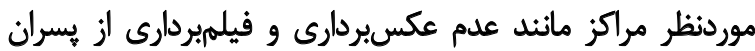

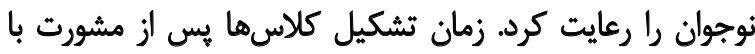

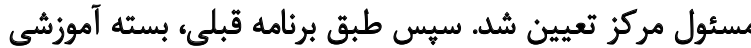

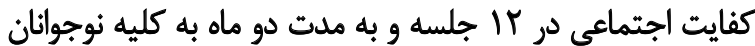

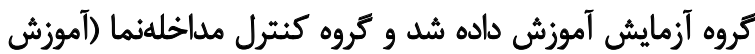

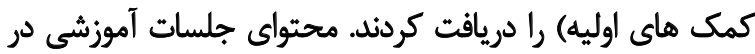

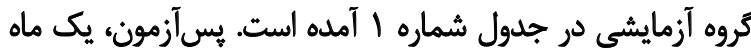

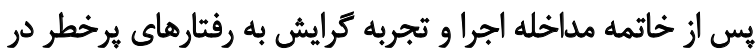

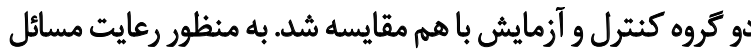

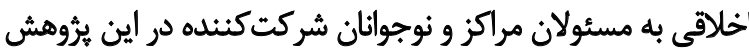

1. Iranian Adolescents Risk-taking Scale (IARS) 
جدول (. عناوين كلى يروتكل جلسات آموزش كفايت اجتماعى

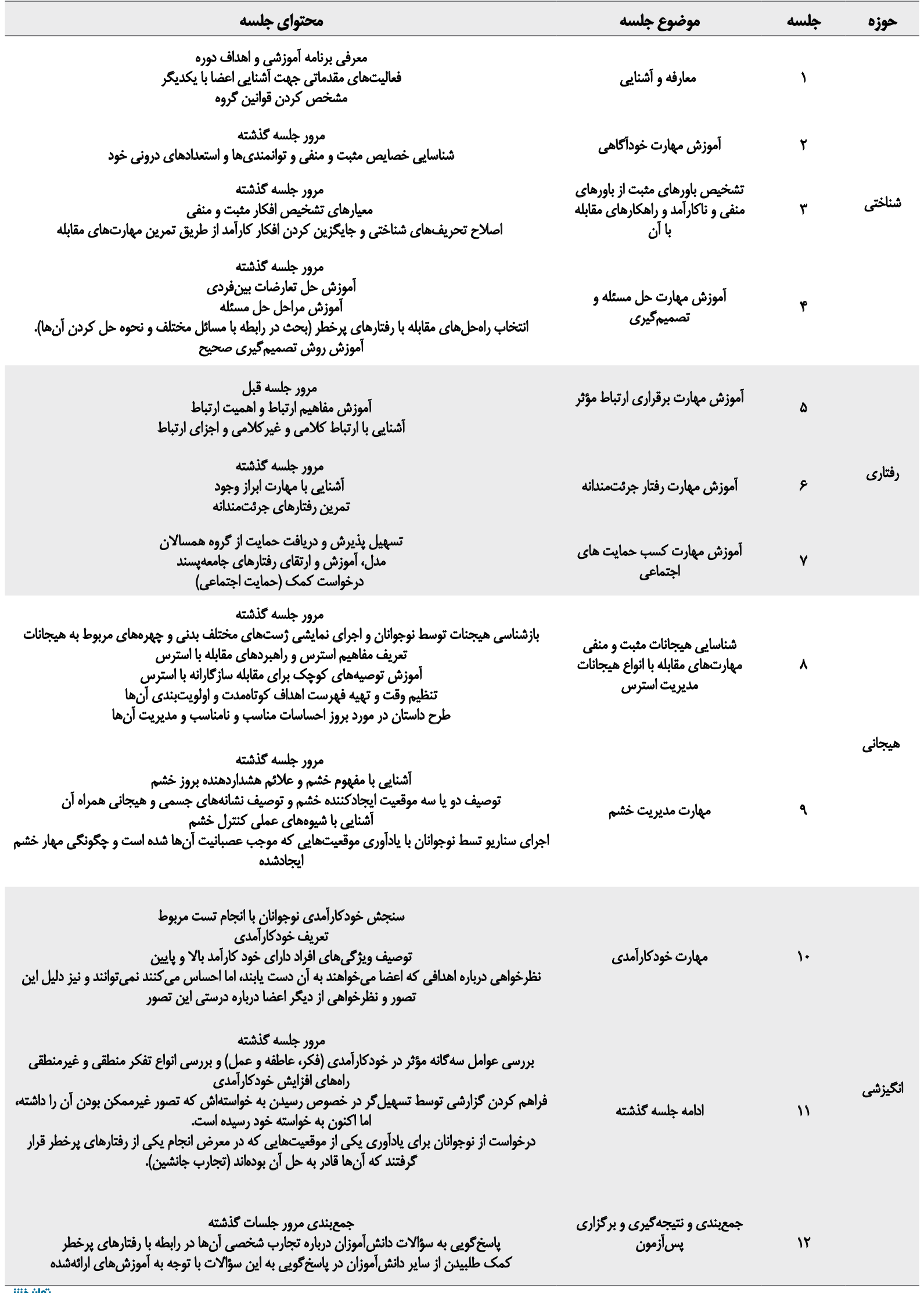


جدول r. ميائكين و انحراف معيار متغيرهاى يثروهش در بيش آزمون و يسآزمون كروه كنترل و آزمايش

\begin{tabular}{|c|c|c|c|}
\hline \multicolumn{2}{|c|}{ كروها } & \multirow{3}{*}{ موقعيت } & \multirow{3}{*}{ متغيرها } \\
\hline 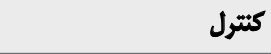 & أزمايش & & \\
\hline ميانكين +انحراف معيار & ميانكين +انحراف معيار & & \\
\hline $\mid \& / N E \pm T / N$ & $\mid Q / N E \pm F / \Delta A$ & ييش آزمهون & \\
\hline $\mid V / N E \pm F / W$ & $\mid r / \pi r \pm r / M$ & يس آزمون & 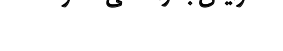 \\
\hline $\mid r / 9 \Psi \pm F / 19$ & $\| F / / H+F / V$ & ييش آزمون & \\
\hline $1 T / M T+M / \Delta q$ & $\| / \Delta r \pm r / \backslash \&$ & يس آزمون & 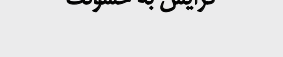 \\
\hline$W / \Delta \Psi+\Psi / \Lambda$. & $W /+\varepsilon \pm \Delta / r \wedge$ & ييش أزمون & \\
\hline$|r / K \varepsilon \pm F / A|$ & $N \Lambda \cdot \pm r / V \Delta$ & يس أزمون & كرايس بيه مصرق سيكار \\
\hline$V V / N E \pm E / P P$ & 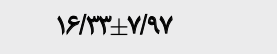 & يبيش أزمون & \\
\hline$W F E \pm \Delta / \Delta V$ & $10 \pm r / M^{\circ}$ & يس أزمون & كرائ به مصرف \\
\hline $\mid \varphi+\varphi / \Delta s$ & $\mid Q / P \cdot \pm A$ & ييش آزمون & 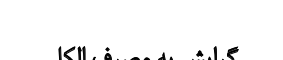 \\
\hline $\mid \Delta / Y \sim \pm \Delta / / \gamma$ & $\| F / M r \pm F / M$ & يس آزّمون & (1) \\
\hline $11 / \pi T \pm r / \pi f$ & $\mid r / K \varepsilon \pm \Delta / M$ & ييش آزمون & \\
\hline $\mid r / r \varepsilon \pm r / F q$ & $1 \cdot / A \cdot \pm r / 9 f$ & يس آزمون & 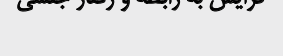 \\
\hline$q / n+4 / 4 q$ & $1 \cdot / N \varepsilon \pm \Delta / \Delta V$ & ييش آزمون & \\
\hline $1.188 \pm r / R$ & $N T F \pm T / N T$ & يسآزعون & 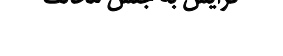 \\
\hline$Q F / T \& \pm T T / 8$. & $1+\circ / q T \pm T / \Delta V$ & ييش آزمون & كرايش به رفتارهاي برخطر (نمره \\
\hline $1 \cdots / 1 \cdot \pm r \cdot / 8 V$ & $|1| / N \varepsilon \pm \mid \varepsilon / \cdot V$ & يسآزمون & \\
\hline
\end{tabular}

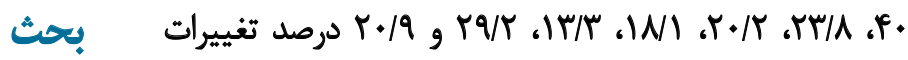

اين يُروهش با هدف بررسى اثربخشى آموزش كفايت اجتماعى

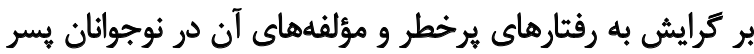

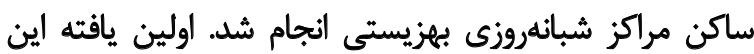

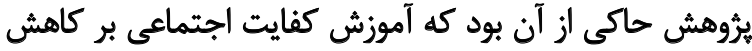

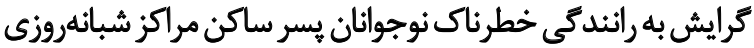

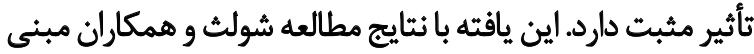

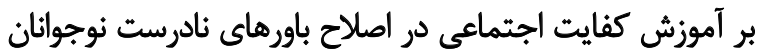

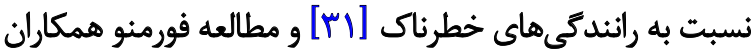

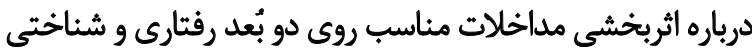

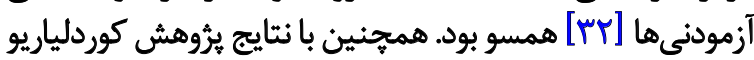

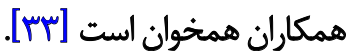

دومين يافته إثوهش مبنى بر اين بود كه آموزش كفايت

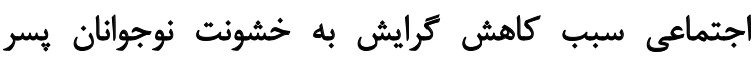

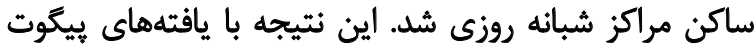

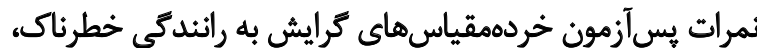

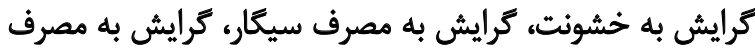

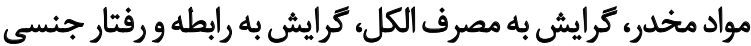
و كرايش به جنس مخالف را تبيين مي كند.

با توجه به نتايج تحليل كوواريانس (جدول شماره ب)، مشخص

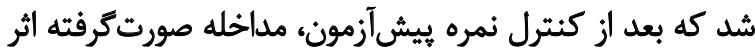

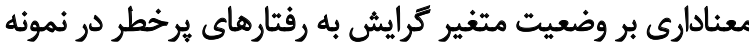

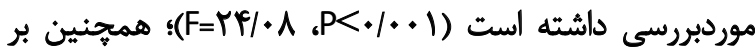

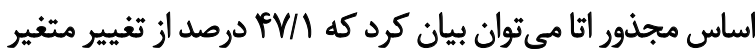

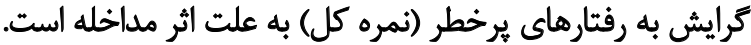

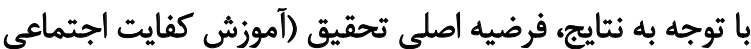

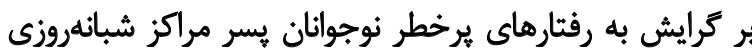

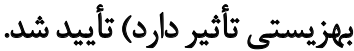


جدول ب. نتايج تحليل كوواريانس جندمتغيره براى تعيين تأثير آموزش كفايت اجتماعى بر كرايش به رفتارهاى يرخطر و مؤلفهاى آن

\begin{tabular}{|c|c|c|c|c|c|c|}
\hline مجذور اتا & P-value & $\mathbf{F}$ & ميانكين مجذورات & درجه أزادى & مجموع مجذورات & متغيرهاى وابسته \\
\hline$\cdot / \mu \cdot r$ & ${ }^{1} \mathrm{p}<\cdot / . .1$ & WIT & $r \Delta T / \Omega$ & 1 & $\operatorname{rar} / / Q$ & كرايش به رائندى خطرناك \\
\hline ATMA & $\% . . v$ & NPY & $P / \Delta r$ & 1 & $P \cdot / A r$ & كرايش به خشونت \\
\hline$\cdot \pi \cdot r$ & .1 .10 & $\varepsilon|A|$ & AN/18 & 1 & $A N / / 8$ & كرايش به مصرف سيكار \\
\hline.$|M|$ &.$/ . r 1$ & $\Delta / q$ & $9 \pi / 91$ & 1 & $94 / 41$ & كرايش به مصرف مواد مخلر \\
\hline 每 & .1 .0 & $\varphi / M F$ & $\mathrm{PW} / \mathrm{MA}$ & 1 & 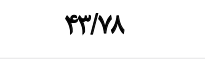 & كرايش به هصرف الكل \\
\hline . /rar & .0 .4 & $11 / 1$ & $\Delta T / M$ & 1 & $\Delta r / T$ & كرايش به رابطله و رقتار جنسى \\
\hline.$/ r \cdot 9$ & ( & V/Ir & $8 . / 4 \pi$ & 1 & $8 . / 4 \pi$ & كرايش به جنس مخالف \\
\hline$. / p r \mid$ & $\mathbf{P}<+/ .+1$ & $r \varphi /+\Lambda$ & TEST/AF & 1 & TSGT/AF & كرايش به رفتارهاى يرخطر (نمره كل) \\
\hline
\end{tabular}

سومين يافته برؤوهش بيانكر آن است كه آموزش كفايت

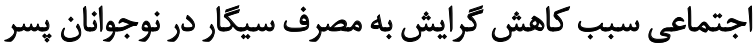

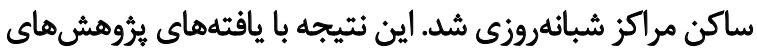

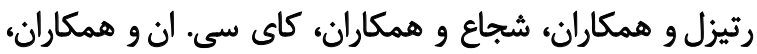

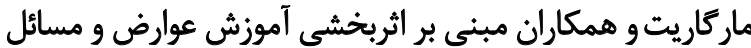

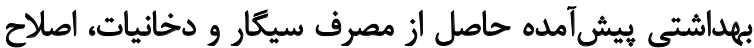

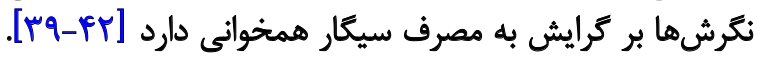

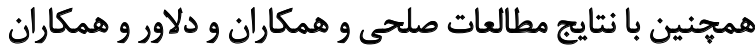

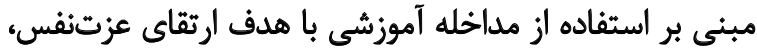

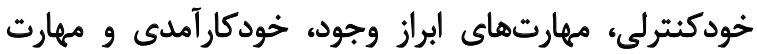

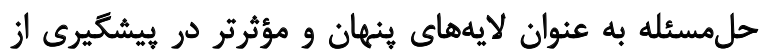

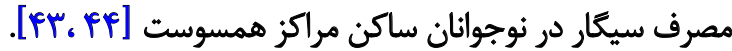
جهارمين يافته اين يُؤوهش حاكى از آن است كه آموزش

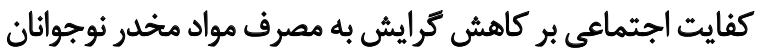

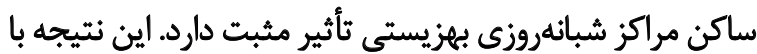

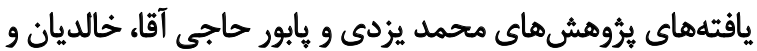

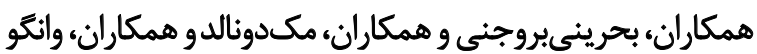

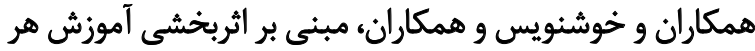

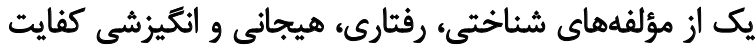

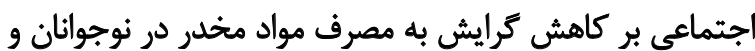

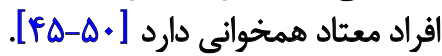

ينجمين يافته برؤهش حاضر بيانكر آن بود كه آموزش

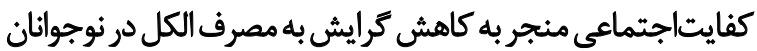

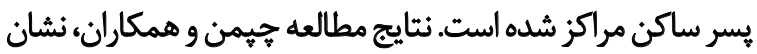

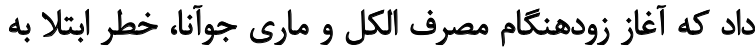

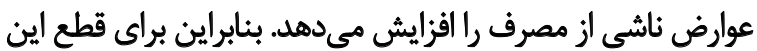

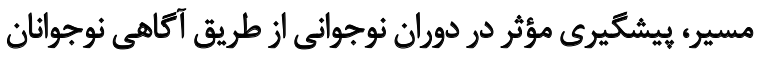
در خصوص الكل و مارى جوآنا، آسيبهائ مرتبط دران با آنان بسيار
و همكاران (1/ (Y) مبنى بر مداخلات مؤثر در جهت كاهش

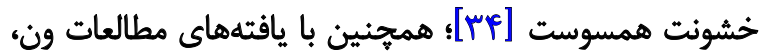
واتكت و همكاران، ماتسون و همكارانش، كايو، دي وال، فين فينكل

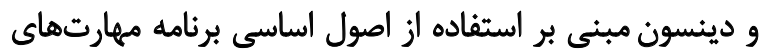

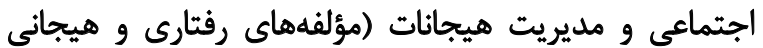

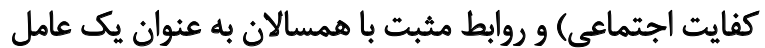

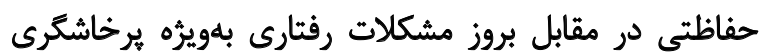

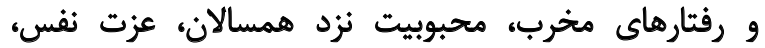

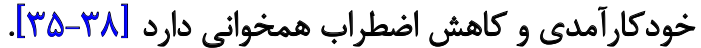
از سوى ديخر، نتيجه مطالعه كاتسى نشان داد كه مداخلات

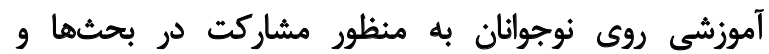

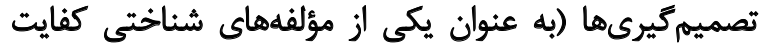

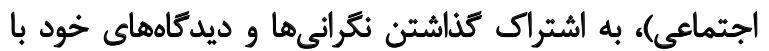

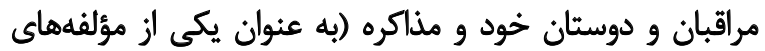

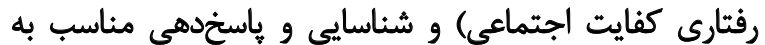

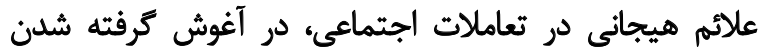

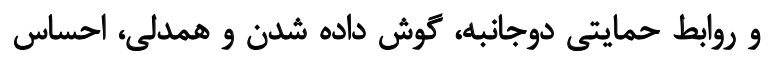

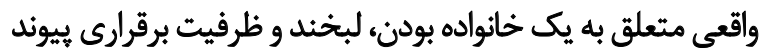

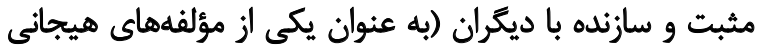

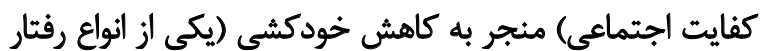

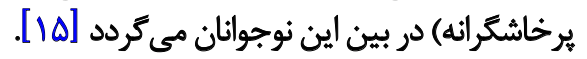

در تبيين اين ياقته ميتوان بيان كرد كه داشتن دانش مربوط

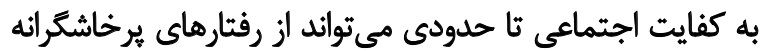

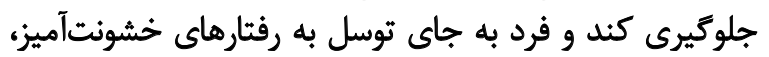

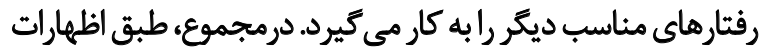

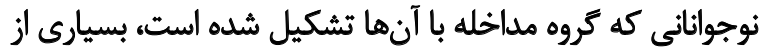

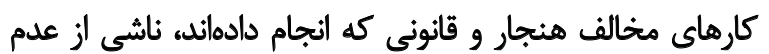
توانايىشان در كنترل خشم بوده است. 
در ثبيين يافتههاى يُؤهش مى تونوان بيان كرد كه كفايت

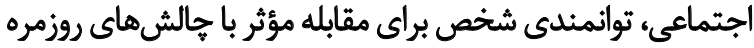

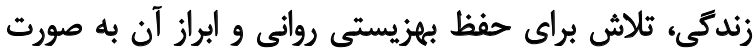

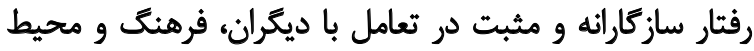

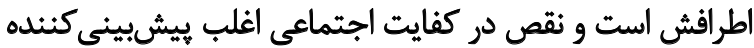

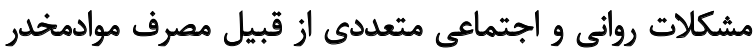

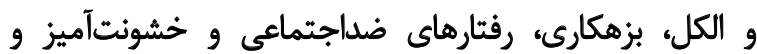

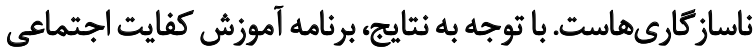

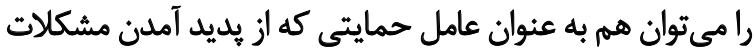

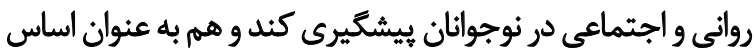
و بايهاى براى رشد سالم آنان به كار برد.

در اين ميان نقش مددكار اجتماعى به عنوان رهبر گروهاز ازئ

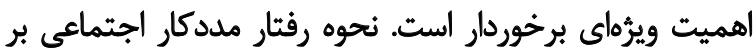

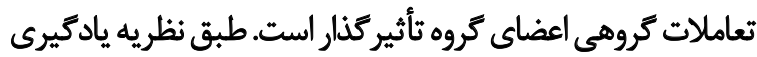

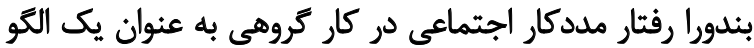

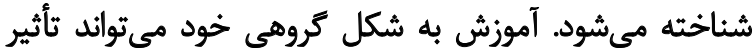

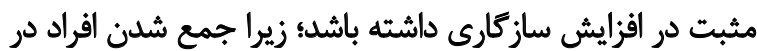

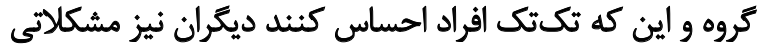

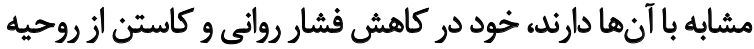

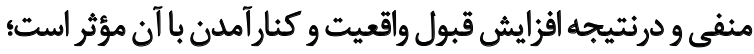

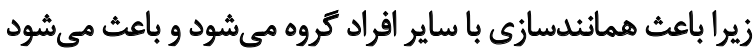

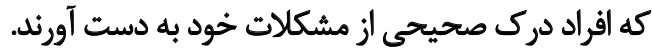

وجود محدوديث در هر بثروهش امرى بديهيى است. در اين

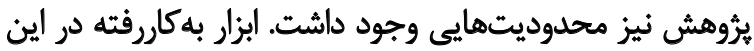

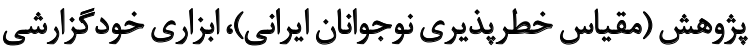

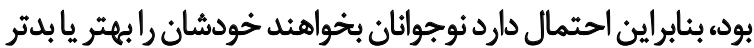

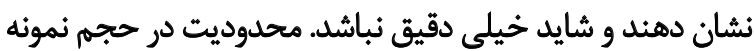

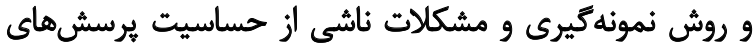

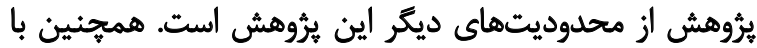

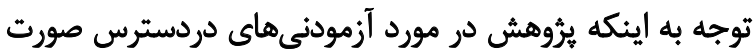

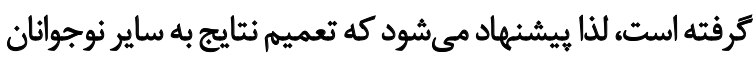
ساكن در مراكز بهزيستى با احتياط صورت كيرد

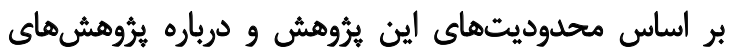

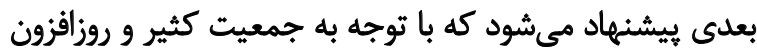

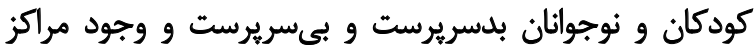

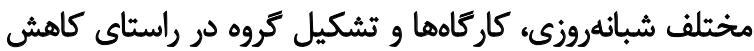

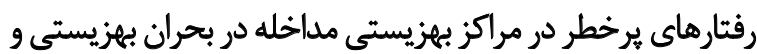

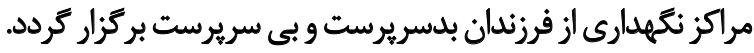

\section{نتيجهيَيرى}

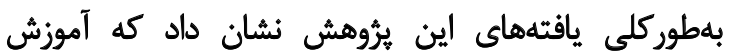

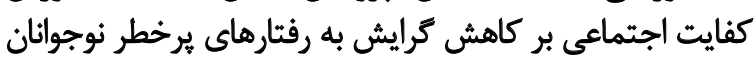
بدسريرست و بىسريرست تأثير درخور توجهي دارئ دارد. كودكان و وخان

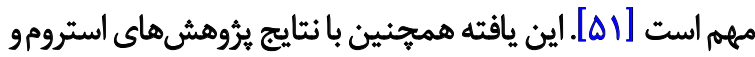

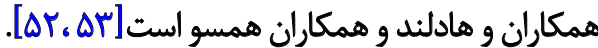

ششمين يافته يرؤهش مبنى بر اين است كه آموزش كفايت

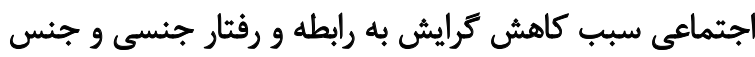

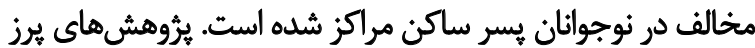

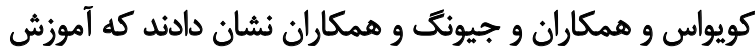

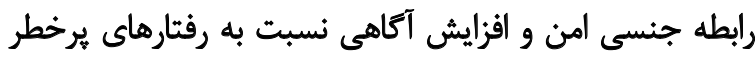

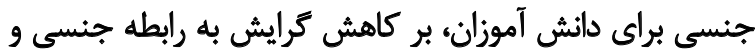

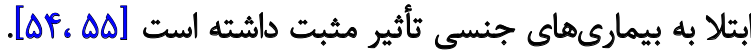

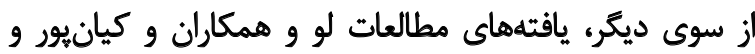

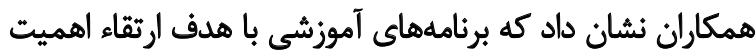

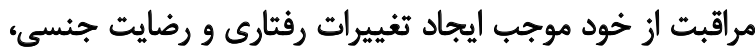

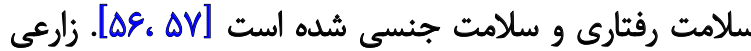

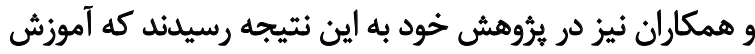

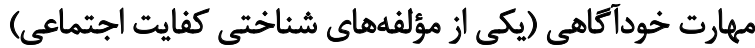
بر كاهش كرايش به جنس مخالف مؤثر است [هئ.

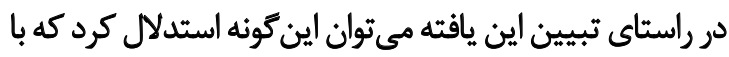

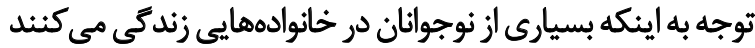

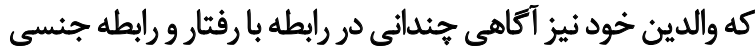

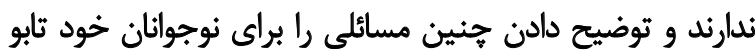

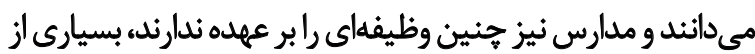

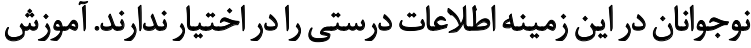

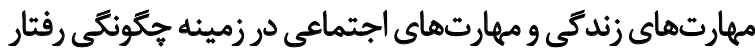

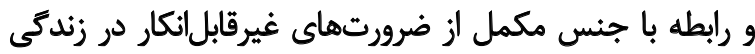

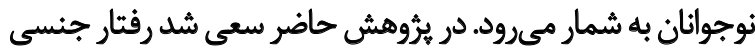

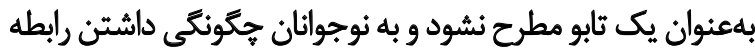

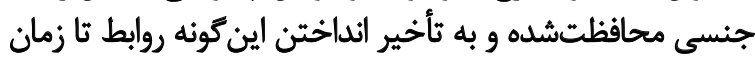

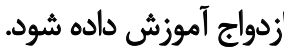

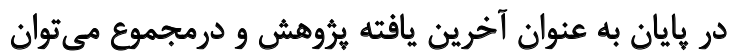

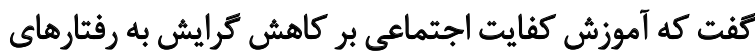

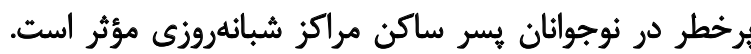

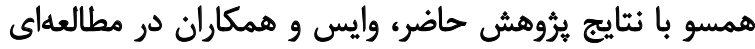

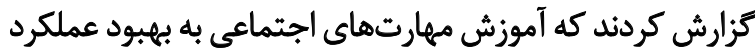

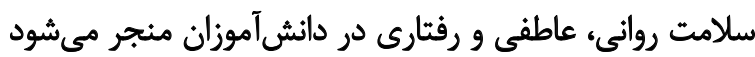

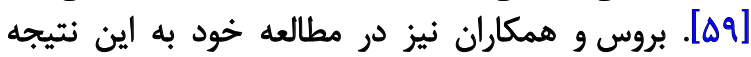

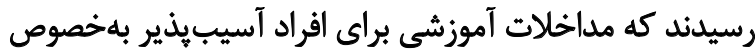

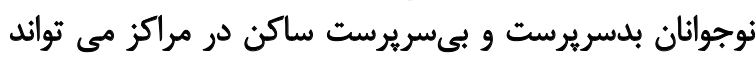

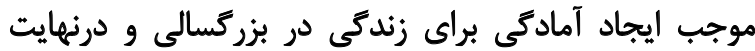

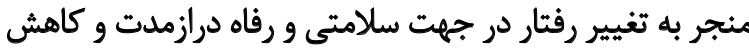

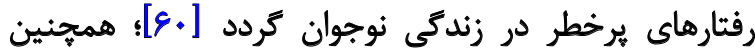

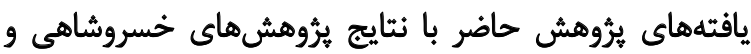

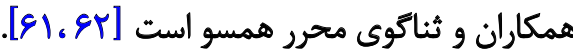




$$
\text { تشيكر وقبر فاني }
$$

نويسندكان مقاله مراتب سياس و قدردانى خود رالز كليه كاركنان

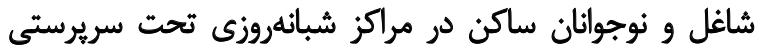

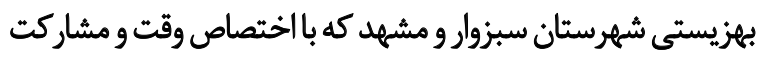
خود كمال همكارى رادر اجراي يثوهش داشتيند، اعلام مي كنيند.

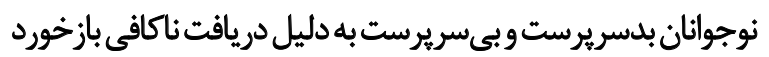

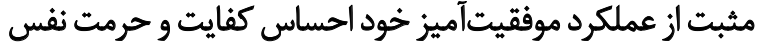

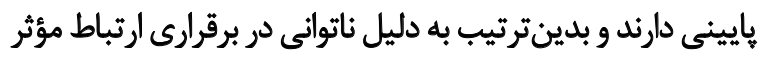

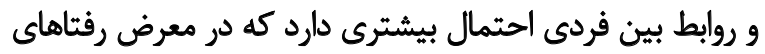

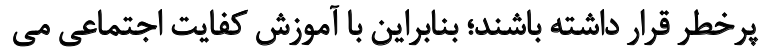

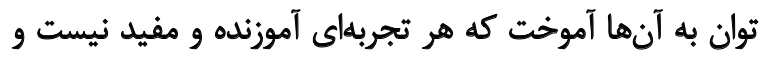

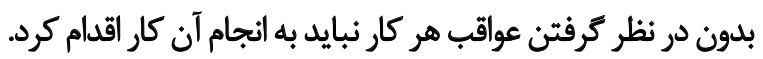

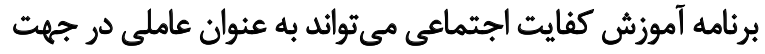

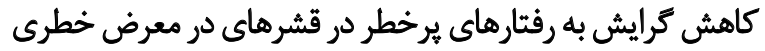

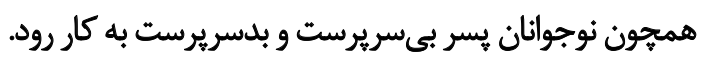

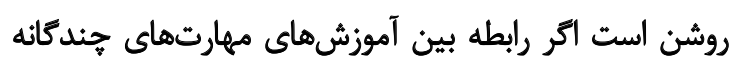

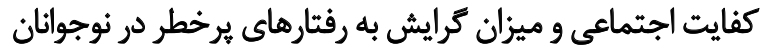

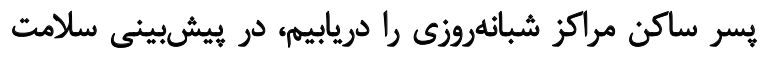

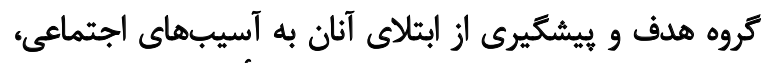

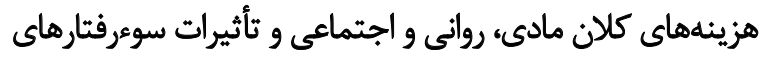
يرخطر بر فرد و جامعه موفقتر خواهي وانيم بود.

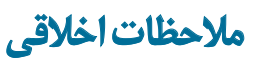

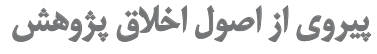

اين بروهش با دريافت كد اخلاقى، مورد تأييد كميته اخلاق

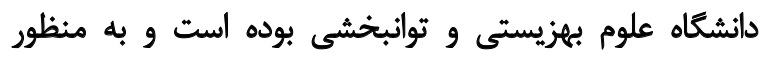

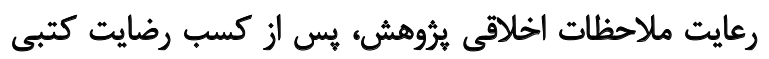

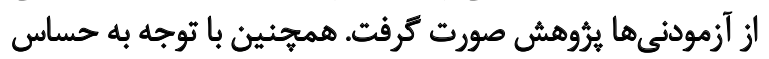

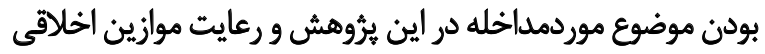

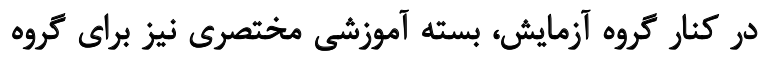

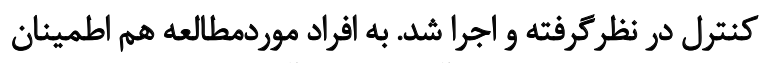

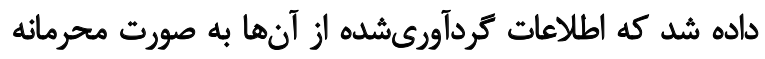

$$
\text { حفظ مى حادود شد مادي }
$$

اين مقاله از ياياننامه كارشناسى ارشد خانم سيما عليزاده در

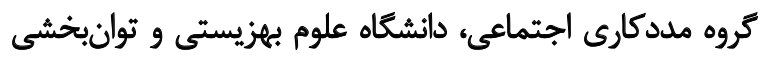

$$
\text { كرفته شده است. }
$$

$$
\text { مشاركت نويسئدكًان }
$$

تحقيق و جمعآورى دادههاى :ثروهش: سيما عليزاده؛

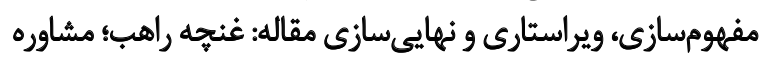
در تنظيم مقاله: زهرا ميرزايى؛ مشاوره در تحليل آمارى: سمانها

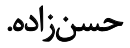

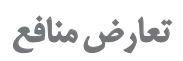

بنابر اظهار نويسندكان اين مقاله تعارض منافع ندارد. 


\section{References}

[1] Rahman W, Mullick M, Pathan MAS, Chowdhury NF, Shahidullah $\mathrm{M}$, Ahmed $\mathrm{H}$, et al. Prevalence of behavioral and emotional disorders among the orphans and factors associated with these disorders. Bangabandhu Sheikh Mujib Medical University Journal. 2012; 5(1):29-34. [DOI:10.3329/bsmmuj.v5i1.10997]

[2] Rashid KH. [Epidemiology of high-risk behaviors among Tehran adolescent girls and boys (Persian)]. Social Welfare. 2015; 15(57):31-55.

[3] Statistical center of iran [Internet]. (Aug 2016) [cited2018]; Available from: https://www.amar.org.ir/

[4] Mirdrikvand F, Ghadampour E, Kavarizadeh M. [The effect of life skills training on psychological capital and adaptability of adolescent girls with irresponsible parents (Persian)]. Quarterly Journal of Social Work. 2016; 5(3):23-30.

[5] Simões C MM, Matos M, Equipa do Projecto Aventura Social. Risk behaviors in adolescents with special needs: Are social and emotional competences important? Procedia - Social and Behavioral Sciences. 2012; 69:2219-27. [DOI:10.1016/j.sbspro.2012.12.191]

[6] Ferreira M, Simoesa C, Matos MG, Ramiro L, Dinez JA, Social Adventure Team. The role of social and emotional competence on risk behaviors in adolescence. International Journal of Emotional Education. 2012; 4(1):43-55.

[7] Slusky, RI. Decreasing high-risk behavior in teens. A theatre program empowers students to reach out to their peers. Health Care Exececutive. 2004; 19(1):48-9.

[8] Ayatmehr F, Khaleghi M, Mohammadkhani S, Ghiasi H. The efficacy of education of adolescent empowerment in prevention of risky behaviors in child labor. Socialworkmag. 2015; 4(1):16-23.

[9] Kann L, McManus T, Harris WA, Shanklin SL, Flint KH, Hawkins $\mathrm{J}$, et al. Youth risk behavior surveillance - United States, 2015. Surveillance Summaries. 2016; 65(6):1-174. [DOI:10.15585/ mmwr.ss6506a1] [PMID]

[10] Khavari Z, Heidari P, Maraghi E, Montazeri MB. [Investigating factors related to risky sexual activity based on the theory of planned behavior among Yazd University Students (Persian)]. The Journal of Toloo-e-Behdasht. 2010; 8(3-4):1-18.

[11] Terzian MA, Andrews KM, Moore KA. Preventing multiple risky behaviors among adolescents: Seven strategies. Research-to-Results Brief. Publication; 2011.

[12] Ombuya BD, Yambo JMO, Omolo TM. Effects of orphanhood on girl-child's access and retention in secondary school education: A case of rongo district, Kenya. International Journal of Academic Research in Progressive Education and Development. 2017. 1(4):114-136. https://pdfs.semanticscholar.org/ be3c/6d3d997cc990eb9dba425f615e8b5a6c7c54.pdf

[13] Assefa A. Prevalence and Factors of Behavioral Disorders among Orphansin Selam and SOS children villages. [MS Thesis]. Addis Ababa: Addis Ababa University; 2015.

[14] Dar MM, Hussain SK, Qadri S, Hussain SS, Fatima SS. Prevalence and pattern of psychiatric morbidity among children living in orphanages of Kashmir. International Journal of Health Sciences and Research (IJHSR). 2015; 5(11):53-60.
[15] Gatsi R. Analysis of the impact of existing intervention programmes on psychosocial needs: Teenage orphans'perceptions. Academic Research International. 2014; 5(1):180.

[16] Dorsey S, Lucid L, Murray L, Bolton P, Itemba D, Manongi R, et al. A qualitative study of mental health problems among orphaned children and adolescents in Tanzania. The Journal of Nervous and Mental Disease. 2015; 203(11):864-70. [DOI:10.1097/NMD.0000000000000388] [PMID] [PMCID]

[17] Jalilabkenar S, Ashoori M, Pourmohamadreza Tajrishi M. [The effectiveness social competence instruction on the adaptation behavior in male students with intellectual disability (Persian)]. Journal of Rehabilitation. 2013; 13(5):104-13.

[18] Nestler J, Goldbeck L. A pilot study of social competence group training for adolescents with borderline intellectual functioning and emotional and behavioural problems (SCT-ABI). Journal of Intellectual Disability Research. 2011; 55(2):231-41. [DOI:10.1111/j.1365-2788.2010.01369.x] [PMID]

[19] Nesayan A, Asadi Gandomani R. [Effectiveness of social skills training on behavioral problems in adolescents with intellectual disability (Persian)]. Journal of Rehabilitation. 2016; 17(2):158-67. [DOI:10.21859/jrehab-1702158]

[20] Felner RD LA, Phillips RS. Social competence and the language of adequacy as a subject matter for psychology: A quadripartite tri-level framework. The Development of Social Competence in Adolescence. 1990; 245-64.

[21] Rockhill CM, Stoep AV, McCauley E, Katon WJ. Social competence and social support as mediators between comorbid depressive and conduct problems and functional outcomes in middle school students. Journal of Adolescence. 2009; 32(3):535-53. [DOI:10.1016/j.adolescence.2008.06.011] [PMID]

[22] Langeveld JH, Gundersen KK, Svartdal F. Social competence as a mediating factor in reduction of behavioral problems. Scandinavian Journal of Educational Research. 2012; 56(4):381-99. [DOI:10.1080/00313831.2011.594614]

[23] Gundersen KK. Reducing behaviour problems in young people through social competence programs. International Journal of Emotional Education. 2010; 2(2):48-62.

[24] Min MO, Minnes S, Kim J-Y, Yoon M, Singer LT. Individual assets and problem behaviors in at-risk adolescents: A longitudinal cross-lagged analysis. Journal of Adolescence. 2018; 64:5261. [DOI:10.1016/j.adolescence.2018.01.008] [PMID] [PMCID]

[25] Haight W, Black J, Sheridan K. A mental health intervention for rural, foster children from methamphetamine-involved families: Experimental assessment with qualitative elaboration. Children and Youth Services Review. 2010; 32(10):114657. [DOI:10.1016/j.childyouth.2010.06.024] [PMID] [PMCID]

[26] Keshtmand Z. [The effectiveness of social competence training on behavioral performance of elementary school girls (Persian)]. [Internet]. 2017[Cited 2020Apr11]. Available from: https://www.civilica.com/Paper-PSCONF05-PSCONF05_130.html

[27] Vahedi SH, Fathiazar S. [The effect of social competence training on decreasing in aggression pre-school boys (Persian)]. The Quarterly Journal of Fundamentals of Mental Health. 2006; 8:131-40. 
[28] Yarahmadiyan N. [Improving individual social abilities and general health of adolescents through enhancement of social competence (Persian)]. International Journal of Behavioral Sciences. 2012; 6(3):279-88

[29] Rezaie E, Haji Alizadeh K. [The compare risky behavior, communication skills and self-efficacy between adolescences of orphanage, replacement families and normal families in Bandar Abbas (Persian)]. Journal of Nursing Education. 2017; 6(5):54-61.

[30] Zadehmohammadi A, Ahmadabadi Z, Heidari M. [Construction and assessment of psychometric features of iranian adolescents risk-taking scale (Persian)]. Iranian Journal of Psychiatry and Clinical Psychology. 2011; 17(3):218-25.

[31] Li L, Shults RA, Andridge RR, Yellman MA, Xiang H, Zhu M. Texting/ emailing while driving among high school students in 35 States, United States, 2015. Journal of Adolescent Health. 2018; 63(6):7018. [DOI:10.1016/j.jadohealth.2018.06.010] [PMID] [PMCID]

[32] Hayashi Y, Foreman AM, Friedel JE, Wirth O. Executive function and dangerous driving behaviors in young drivers. Transportation Research Part F: Traffic Psychology and Behaviour. 2018; 52:51-61. [DOI:10.1016/j.trf.2017.11.007] [PMID] [PMCID]

[33] Cordellieri P, Baralla F, Ferlazzo F, Sgalla R, Piccardi L, Giannini AM. Gender effects in young road users on road safety attitudes, behaviors and risk perception. Frontiers in Psychology. 2016; 7:1412. [DOI:10.3389/fpsyg.2016.01412] [PMID] [PMCID]

[34] Piggott T HD, Mann R, Hamilton HA, Donnelly PD, Manson $\mathrm{H}$. Youth violence victims and perpetrators in Ontario: Identifying a high-risk group and a focus for public health prevention. Canadian Journal of Public Health. 2018 ;109(2):195-203. [DOI:10.17269/s41997-018-0061-6] [PMID] [PMCID]

[35] Van Vugt ES, Deković M, Prinzie P, Stams GJJM, Asscher JJ. Evaluation of a group-based social skills training for children with problem behavior. Children and Youth Services Review. 2013; 35(1):162-7. [DOI:10.1016/j.childyouth.2012.09.022]

[36] Matson L, Fodstad CJ, Rivert TT. The relationship of social skills and problem behaviors in adults with intellectual disability and autism or PDD-NOS. Research in Autism Spectrum Disorders. 2009; 3(1):258-68. [DOI:10.1016/j.rasd.2008.07.001]

[37] Koiv K. Social skills training as a mean of improving intervention for bullies and victims. Procedia - Social and Behavioral Sciences. 2012; 45:239-46. [DOI:10.1016/j.sbspro.2012.06.560]

[38] DeWall CN, Finkel EJ, Denson TF. Self-control inhibits aggression. Social and Personality Psychology Compass. 2011; 5(7):458-72. [DOI:10.1111/j.1751-9004.2011.00363.x]

[39] Okuyemi KS, Reitzel LR, Fagan P. Interventions to reduce tobacco-related health disparities. Nicotine \& Tobacco Research. 2015; 17(8):887-91. [DOI:10.1093/ntr/ntv096 PMid:26180213 PMCid:PMC4502764] [PMID] [PMCID]

[40] Shuja M, Sarrafzadegan N, Roohafza HR, Sadeghi M, Ghafari $M$, Mohammadian $M$, et al. Factors associated with cigarette smoking in central parts of Iran. Asian Pacific Journal of Cancer Prevention. 2017; 18(3):647-53.

[41] Onyechi KCN, Eseadi C, Umoke PCI, Ikechukwu-Ilomuanya AB, Otu MS, Obidoa JC, et al. Effects of a group-focused cognitive behavioral health education program on cigarette smoking in a sample of Nigerian prisoners. Medicine. 2017; 96(1):e5158. [DOI:10.1097/MD.0000000000005158] [PMID] [PMCID]

[42] Cousson-Gelie F, Lareyre O, Margueritte M, Paillart J, Huteau $\mathrm{ME}$, Djoufelkit $\mathrm{K}$, et al. Preventing tobacco in vocational high schools: Study protocol for a randomized controlled trial of $\mathrm{P} 2 \mathrm{P}$, a peer to peer and theory planned behavior-based program. BMC Public Health. 2018; 18(1):494. [DOI:10.1186/s12889-018 5226-y PMid:29653527 PMCid:PMC5899383] [PMID] [PMCID]

[43] Solhi M, Abasi H, Hazavehei MM, Roshanaei GH. [Effect of educational intervention on empowerment of high school student in prevention of smoking (Persian)]. Razi Journal of Medical Sciences. 2014; 21(118):52-63.

[44] Delavar A, Rashid KH. The effect of teaching expression and problem solving skills in preventing and reducing smoking in Tehran students. Journal of Psychological Studies. 2012; 1(1):31-40. [DOI:10.22051/PSY.2004.1654]

[45] Mohammadi-Zeydi I, Pakpour-Hagiagha A. [Application of the theory of planned behavior for the prevention of substance abuse among male adolescents (Persian)]. Iranian Journal of Health Education and Health Promotion. 2016; 3(4):298-310.

[46] Khaledian M, kamarzarin M, Jalalian A. [The effectiveness of group cognitive behavior therapy on reduction of addicts' depression (Persian)]. Quarterly Journal of Research on Addiction 2014; 8(29):77-88.

[47] Bahreini-Borujeni M, Ghaedamini-Harouni GH, Saedzadeh HR, Sepehri-Borujeni K. [Examination of the four effectiveness methods of preventive from drug abuse on changing attitude towards drug abuse in the high schools boys (Persian)]. Journal of Shahrekord University of Medical Sciences. 2014; 16(2):43-51.

[48] Popova L, McDonald EA, Sidhu S, Barry R, Richers Maruyama TA, Sheon NM, et al. Perceived harms and benefits of tobacco, marijuana, and electronic vaporizers among young adults in Colorado: Implications for health education and research. Addiction. 2017; 112(10):1821-9.

[49] Wang YC, Yu JC, Chen JY, Wang ZZ. Factors associated with illicit drug use intention in secondary vocational school students based on theory of triadic influence. Zhonghua liu xing bing xue za zhi = Zhonghua liuxingbingxue zazhi. 2018; 39(7):925-30.

[50] Mohammad-Najar N, Khoshnevis E, Banisi P. Effectiveness of positive thinking skills training on the hope and quality of life of drug-dependent people. Addiction \& Health. 2017; 9(3):120-8.

[51] Newton NC, Chapman C, Slade T, Conroy C, Thornton L, Champion KE, et al. Internet-based universal prevention for students and parents to prevent alcohol and cannabis use among adolescents: Protocol for the randomized controlled trial of climate schools plus. JMIR Research Protocols. 2018; 7(8):e10849. [DOI:10.2196/10849] [PMID] [PMCID]

[52] Strom HK, Adolfsen F, Handegard BH, Natvig H, Eisemann M, Martinussen $\mathrm{M}$, et al. Preventing alcohol use with a universal school-based intervention: Results from an effectiveness study. BMC Public Health. 2015; 15(1):337. [DOI:10.1186/ s12889-015-1704-7] [PMID] [PMCID]

[53] Hadland SE, Knight JR. Brief interventions for alcohol use: Where, when, and how? Pediatrics. 2015; 136(4):e1002e1004. [DOI:10.1542/peds.2015-2713] [PMID] [PMCID] 
[54] Doubova SV, Martinez-Vega IP, Infante-Castaneda C, PerezCuevas R. Effects of an internet-based educational intervention to prevent high-risk sexual behavior in Mexican adolescents. Health Education Research. 2017; 32(6):487-98 [DOI:10.1186/ s12889-016-2990-4] [PMID] [PMCID]

[55] Jeong S, Cha C, Lee J. The effects of STI education on Korean adolescents using smartphone applications. Health Education Journal. 2017; 76(7):775-86. [DOI:10.1177/0017896917714288]

[56] Lou CH, Wang XJ, Tu XW, Gao ES. Impact of life skills training to improve cognition on risk of sexual behavior and contraceptive use among vocational school students in Shanghai, China. Journal of Reproduction and Contraception. 2008; 19(4):23951. [DOI:10.1016/S1001-7844(08)60026-7]

[57] Boroumandfar Z, Kianpour M, Zargham A, Abdoli S, Tayeri $\mathrm{K}$, Salehi $\mathrm{M}$, et al. Changing beliefs and behaviors related to sexually transmitted diseases in vulnerable women: A qualitative study. Iranian Journal of Nursing and Midwifery Research. 2017; 22(4):303-7. [DOI:10.4103/ijnmr.IJNMR_117_16] [PMID] [PMCID]

[58] Zarie F, Khakbaz H, Karami H. [Determining the effectiveness of self-awareness skill training on reducing high-risk behaviors associated with the opposite sex addict (Persian)]. Journal of Research on Addiction. 2010; 15(4):63-70.

[59] Dang HM, Weiss B, Nguyen CM, Tran N, Pollack A. Vietnam as a case example of school-based mental health services in low and middle income countries: Efficacy and effects of risk status. School Psychology International. 2017; 38(1):22-41. [DOI:10.1177/0143034316685595] [PMID] [PMCID]

[60] Burrus BB, Krieger K, Rutledge R, Rabre A, Axelson S, Miller $A$, et al. Building bridges to a brighter tomorrow: A systematic evidence review of interventions that prepare adolescents for adulthood. American Journal of Public Health. 2018; 108(S1):S25-S31. [DOI:10.2105/AJPH.2017.304175] [PMID] [PMCID]

[61] Bahadorikhosroshahi J, Habibikaleybar R, Farid A. [The effect of education on health-promoting with academic stress, life skills and risky behaviors among students (Persian)]. Education Strategies in Medical. 2017; 10(1):64-73.

[62] Sanagouye Moharer GH, Firozkohi B, Nazari R. [The effectiveness of promoting health education program on opinion and risky behaviors among students (Persian)]. Contemporary Psychology. 2017; 12(Suppl.):451-5; Available from: https://www. civilica.com/Paper-CIPSA06-CIPSA06_323.html 
This Page Intentionally Left Blank 